\title{
Experiment on space charge driven nonlinear resonance crossing in an ion synchrotron
}

\author{
G. Franchetti, O. Chorniy, I. Hofmann, W. Bayer, F. Becker, P. Forck, T. Giacomini, M. Kirk, T. Mohite, \\ C. Omet, A. Parfenova, and P. Schütt \\ GSI Darmstadt, D-64291 Darmstadt, Germany
}

(Received 18 August 2009; published 19 November 2010)

\begin{abstract}
Trapping of particles in nonlinear resonances in the presence of space charge and synchrotron motion may be a source of beam halo generation and beam loss in high intensity synchrotrons, in particular for extended storage times at the injection plateau as planned for the SIS100 synchrotron of the FAIR project. Although extensive simulation studies have theoretically demonstrated this mechanism, experimental evidence was so far limited to demonstration experiments at the CERN Proton Synchrotron (PS) in 20022003 using an octupolar resonance. Here we describe new experiments at the SIS18 synchrotron at GSI, where the resonance is driven by a sextupolar field error and horizontal static tune scans are taken across the resonance stop band. The new data significantly extend the previous observations by a complete set of measurements comparing beams with and without rf, both at low and high intensity. The correlation between transverse beam loss and simultaneous bunch length shortening provides strong evidence that the measured emittance and the loss in intensity are indeed caused by periodic resonance crossing, leading to the main effect of scattering but also to a lesser extent to the trapping of particles due to the combined effect of the nonlinear resonance and the space charge.
\end{abstract}

DOI: 10.1103/PhysRevSTAB.13.114203

PACS numbers: 41.75.-i, 29.27.Bd, 05.45.Pq

\section{INTRODUCTION}

The dynamics of particle beams in circular accelerators is naturally subject to the periodicity of the sequence of elements composing an accelerator. The $N$ th harmonics of a distribution of nonlinear errors of $n_{0}$ th order excites the resonances $n Q_{x}+m Q_{y}=N$, where $Q_{x}, Q_{y}$ are the accelerator tunes, and $n+m=n_{0}$ [1]. Although resonances should be avoided, it may happen that during machine operations unwanted drifts of $Q_{x}, Q_{y}$ might lead to a resonance crossing as, for example, was found in the Fermilab Booster [2-4]. The beam dynamics for time varying tunes crossing a resonance is complex, and has been addressed in several pioneering studies in Refs. [5-7]. When the machine tunes cross a stable resonance, the dynamics of a particle have two distinct patterns [8]: (1) If the tunes cross the resonance slow enough (adiabatically), the resonance captures the particle and stays "locked" to it. The consequence is that a particle gains large amplitudes (see also Ref. [9]). (2) If the tunes cross the resonance fast enough, beam particles will receive a small kick by the stable islands and each single particle invariant will be "scattered." The distinction between trapping and scattering regimes can be made via an adiabaticity parameter $T=\left[\partial\left|\vec{x}_{f}(n)\right| / \partial n\right] /\left(Q_{\text {isl. }} \Delta \bar{x}\right)$, where $\vec{x}_{f}$ is the position of the fixed points, $Q_{\text {isl. }}$ is the secondary tune (island tune), and $\Delta \bar{x}$ the island size. This definition is equivalent to that used in Ref. [6]. If $T<1$ the crossing regime is adiabatic and trapping can take place. Experimental studies with low intensity beams on trapping of particles in stable islands are reported in Refs. [10-15]. The notion of transverse trapping or scattering of particles in bunched beams is possible because in synchrotrons the longitudinal motion typically has a frequency much smaller than those in the transverse plane, therefore the longitudinal motion can be treated as a parameter. This approximation is said of the frozen system as the tunes experienced by a beam particle are instantaneous tunes.

Trapping and scattering phenomena become relevant for a repeated crossing of the same resonance, which may easily happen for a bunched beam when the chromaticity is left uncompensated. However, it has recently been realized that space charge also plays a similar role [16]. In a stationary bunch created by a single rf harmonics the longitudinal particle motion, via space charge, changes the transverse tunes of the frozen system as happens with the chromaticity. The relevant feature of the space charge is that it creates a strong nonlinear transverse amplitude dependent detuning, which drives the transverse islands much further out in the phase space than the chromaticity alone. Examples of trapping and scattering phenomena induced by space charge are discussed in Ref. [17] where a numerical study shows how the space charge affects $T$ along a bunch. When space charge is considerable (e.g. $\left.\Delta Q_{x, \text { sc }} \sim 0.2\right)$, and a bunched beam is stored for a long time ( $10^{5}$ or more turns), a periodic resonance crossing increases the transverse amplitude of particles when their tunes are above closer to the resonance. If particles will move within the accelerator acceptance, an emittance increase appears (for small halo radii), otherwise a slow progressive beam loss will characterize the storage. The beam loss created by this mechanism is typically found for tunes above the resonance periodically crossed. In the case of simultaneous presence of space charge and chromaticity, 
the full scenario is even more complicated. As discussed in Ref. [17] the beam loss region may be enlarged up to the chromatic tune spread: In fact, the bunch particles have an effective tune of $Q_{x / y}+\xi_{x / y} Q_{x / y} \delta p / p$, with $\xi_{x / y}$ the normalized horizontal/vertical machine chromaticity, and $\delta p / p$ the particle off momentum. Hence, in spite $Q_{x}, Q_{y}$ may be far from a resonance, several bunch particles are brought periodically close to the resonance by the chromaticity so that their transverse motion is periodically effected by space charge and resonance. An important aspect of the periodic resonance crossing for high intensity bunched beams consists in the induced correlation between beam loss and longitudinal beam size: When the accelerator is tuned close to a resonance (and above it), only particles with large synchrotron amplitude may cross the resonance and therefore become trapped or scattered into a halo and eventually be lost. The correlation between "beam loss" and "bunch shortening" is a strong indication that only particles at large longitudinal amplitude will be lost.

The experimental verification of these high intensity effects, when driven by a controlled 4th order resonance, was carried out at the CERN PS in the years 2002-2003 [18]. The experiment was performed with uncompensated chromaticity creating a "mixed" regime of periodic resonance crossing induced by both the space charge and the chromatic detuning. In spite of this complication, the PS benchmarking experiment has proven that the measured emittance growth and bunch shortening can be interpreted in the frame of a trapping and scattering theory [19]. While these basic mechanisms have been identified on the theoretical and numerical side, a thorough experimental investigation that includes the bunch shortening correlation and the comparison with a coasting beam was still missing. In the experiment described in this paper we attempt to fill this gap. At GSI we have carried out in the SIS18 synchrotron an experimental campaign in which the long-term space charge effect in the presence of a 3rd order resonance (octupoles are not available) was studied. These studies find direct application in the SIS100 synchrotron part of the FAIR project [20-22]: In the main scenario of SIS100 operations, bunched beams of $\mathrm{U}^{28+}$ are stored for $\simeq 1 \mathrm{~s}$ and beam control or containment of beam loss becomes essential: Beam loss above the $\sim 5 \%$ level may trigger an avalanche vacuum degradation process due to the high $\mathrm{U}^{28+}$ desorption yield, which dramatically reduces beam lifetime [23]. The plan of the paper is the following: In Sec. II we discuss the experimental setup, i.e., the experimental conditions in which all our measurements have been performed as well as how we interpret the measurements from the ionization profile monitor (IPM). Section III presents a series of measurements apt to distinguish the several sources affecting the beam quality such as chromaticity, dispersion, and the synchrotron motion. The experimental results are presented and compared with multiparticle simulations. In Sec. IV we discuss how these findings may form a possible proof of principle for high intensity driven trapping/scattering mechanisms acting during long-term storage. Section $\mathrm{V}$ is devoted to the conclusion, and in the Appendix we discuss how we modeled the nonlinear lattice of SIS18 as well as the methodology for assigning error bars.

\section{EXPERIMENTAL SETUP}

\section{A. Beam condition}

In the experimental campaigns an ion beam of ${ }^{40} \mathrm{Ar}^{18+}$ was used. In Fig. 1 is shown an example of the beam emittances measured at the exit of the linac (UNILAC), here with $\epsilon_{x}=6.58 \mathrm{~mm} \mathrm{mrad}, \epsilon_{y}=3.49 \mathrm{~mm} \mathrm{mrad}$. The emittances are computed here by using a cut phase space at $90 \%$ of the peak density, and these numbers refer to the $2 \sigma$ emittance [i.e. to the equivalent Kapachinsky-Vladimirsky (KV) edge]. The beam intensity in the UNILAC was set to $I_{U}=0.8 \mathrm{~mA}$, the injection energy in the SIS18 and other beam and accelerator parameters are reported in Table I. A schematic of SIS18 is shown in Fig. 2. For the purpose of

a)

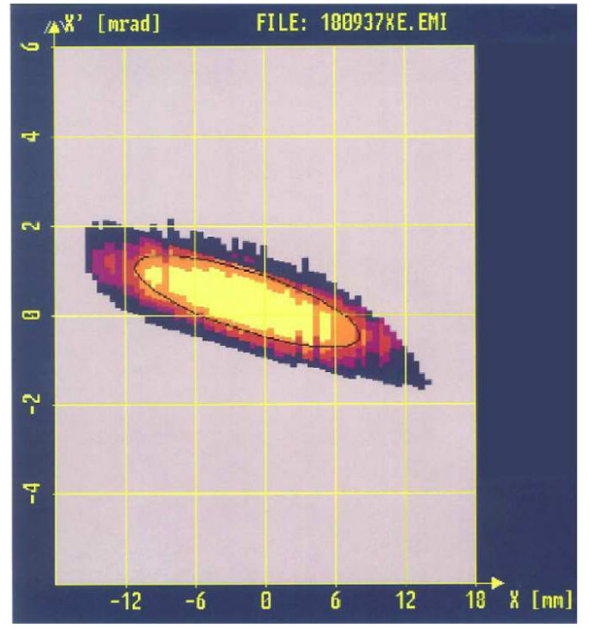

b)

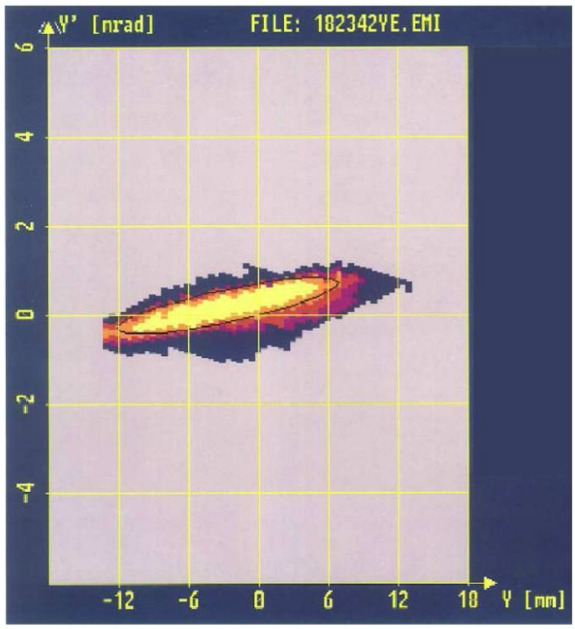

FIG. 1. Horizontal (a) and vertical (b) beam phase space measured in the transfer channel between UNILAC and SIS18. 
TABLE I. Typical parameters of SIS18 and of the ion beam used.

\begin{tabular}{lcc}
\hline \hline Parameter & Value & Units \\
\hline Energy per nucleon & 11.28 & $\mathrm{MeV} / \mathrm{u}$ \\
Ion mass number & 40 & \\
Ion charge state & 18 & \\
rf harmonics & 4 & $\mathrm{kV}$ \\
rf voltage & 4 & \\
Total particles per bunch & $3.125 \times 10^{8}$ & \\
Gamma transition & 5.01 & $\mathrm{Tm}$ \\
Rigidity & 1.077 & $\mathrm{~m}$ \\
SIS18 circumference & 216.1 & $\mathrm{~m}$ \\
Average $\beta_{x}$ & $\sim 8$ & $\mu \mathrm{s}$ \\
Average $\beta_{y}$ & $\sim 10$ & \\
Revolution time & 4.673 & \\
Eta transition & 0.9362 & \\
Synchrotron tune & $6.915 \times 10^{-3}$ & $\mathrm{~ns}$ \\
Bunching factor & 0.3357 & \\
Rms momentum spread & $1.3 \times 10^{-3}$ & $\mathrm{~mm} \mathrm{mrad}$ \\
Bunch length $4 \sigma$ & 560 & $\mathrm{~mm} \mathrm{mrad}$ \\
Maximum $\delta p / p$ in the bucket & $7.4 \times 10^{-3}$ & \\
Horizontal emittance at $2 \sigma$ & 19 & \\
Vertical emittance at $2 \sigma$ & 14 & \\
Horizontal peak tune shift & $-4 \times 10^{-2}$ & \\
Vertical peak tune shift & $-4.5 \times 10^{-2}$ & \\
\hline \hline
\end{tabular}

observing a high intensity driven beam blowup, a beam smaller than the SIS18 acceptances $\left(A_{x} \simeq 200 \mathrm{~mm} \mathrm{mrad,}\right.$ $A_{y} \simeq 50 \mathrm{~mm} \mathrm{mrad}$ ) was created by setting the injection chopper window to $10 \mu$ s over $160 \mu$ s for complete acceptance filling. After injection the beam was stored for $\simeq 1 \mathrm{~s}$ at injection energy, and then accelerated for extraction.

At injection energy the revolution time is $4.6 \mu$ s and an injection with a chopper window of $10 \mu$ s corresponds to two turns equivalent to $2.1 \times 10^{9}$ ions in SIS18. However,

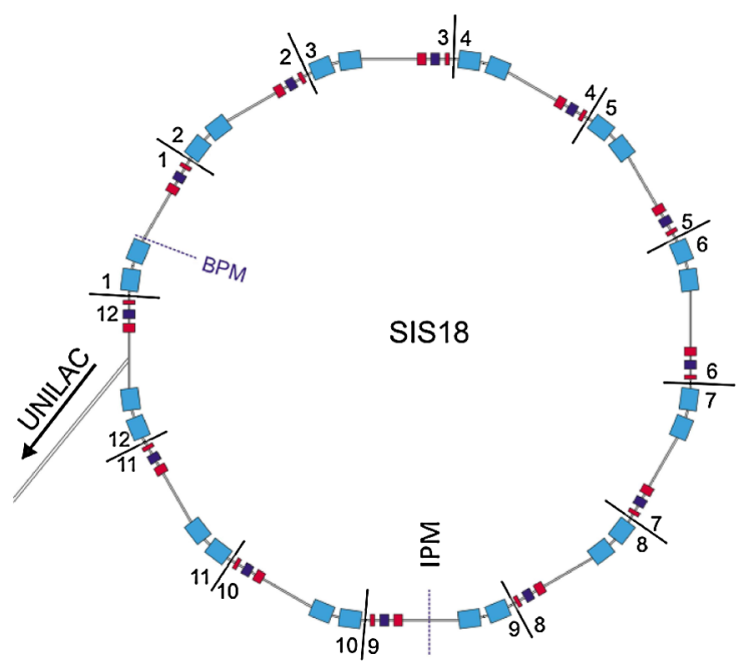

FIG. 2. Layout of the SIS18 and location of the diagnostic devices used. because of the inefficiency of the multiturn injection, the number of particles stored in the SIS18 did not exceed $\sim 1.5 \times 10^{9}$. The beam emittances have been computed from the beam profiles measured with the IPM [24]. In Fig. 3 we plot an example of the beam profiles for a low intensity coasting beam measured right after multiturn injection, and after storage of $1 \mathrm{~s}$ for the tunes $Q_{x}=$ 4.3605, $Q_{y}=3.245$. These tunes are not those set into the SIS18 control system, which are unavoidably affected by systematic shifts, but the corrected tunes. We present throughout this paper tunes already corrected and discuss in Sec. III A the method used to correct the systematic tune shift. The transverse emittances are calculated using the measured distributions and beta functions. The measurements from the IPM have to be interpreted carefully. In fact, the machine acceptance at the position of the IPM provides an area accessible to the beam within $\pm 35 \mathrm{~mm}$ horizontally and $\pm 20 \mathrm{~mm}$ vertically. Consequently, an apparent ion detection measured at $y=40 \mathrm{~mm}$ in Fig. 3(a) cannot be attributed to the ion beam. The reason for this spurious signal is that the IPM spatial area of
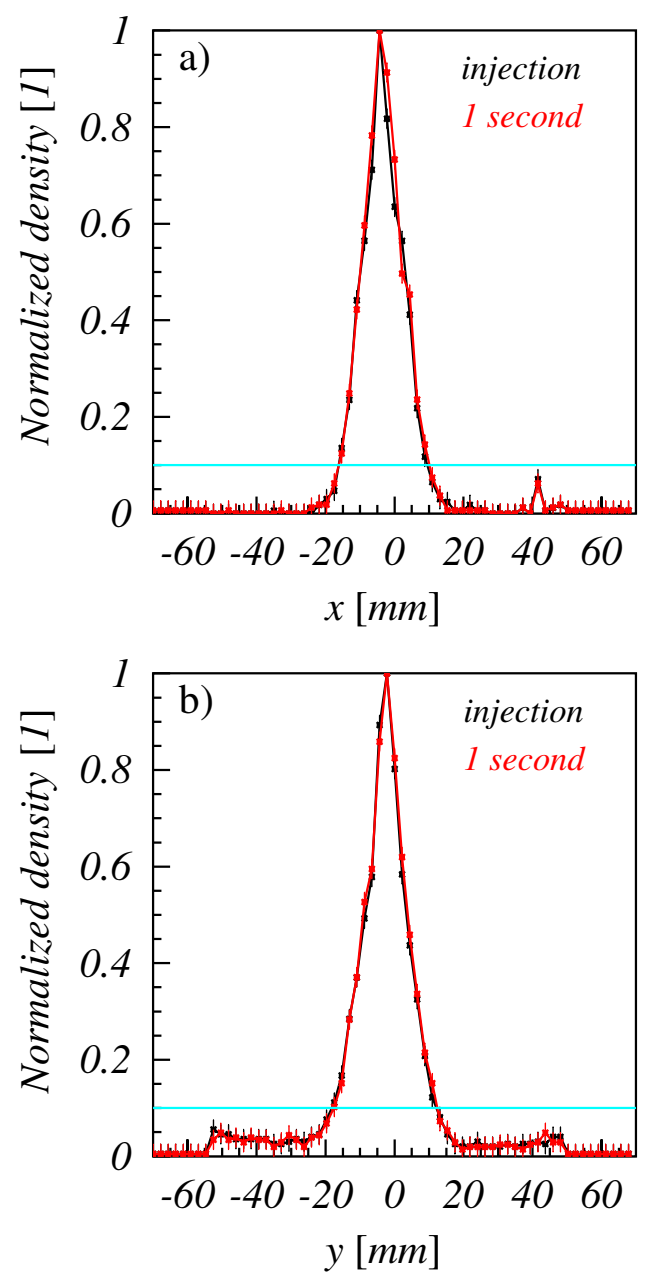

FIG. 3. Horizontal (a) and vertical (b) beam profile measurement for the SIS18 tunes $Q_{x}=4.340, Q_{y}=3.245$. 
detection is larger than the accelerator acceptances. We avoid this artifact by cutting profile data below $10 \%$ of their maximum [blue line in Figs. 3(a) and 3(b)]. Clearly this procedure reduces the effective rms size: For example, for a Gaussian distribution this is a reduction of 9.3\% which causes an emittance reduction of $18 \%$. In calculating the emittances we take into account these corrections (see Appendix B). From Fig. 3 we obtain then the rms beam emittances $\epsilon_{x, \text { rms }} \simeq 5 \mathrm{~mm} \mathrm{mrad}, \epsilon_{y, \text { rms }} \simeq 5 \mathrm{~mm} \mathrm{mrad}$, with edges (at $3 \sigma$ ) corresponding to the emittances: $\epsilon_{x, \text { edge }}=$ $45 \mathrm{~mm} \mathrm{mrad}, \epsilon_{y, \text { edge }}=45 \mathrm{~mm} \mathrm{mrad}$. Therefore the tail of this beam is not at the edge of the SIS18 horizontal/vertical acceptance and possible beam blowup can occur without beam loss. The emittance measurements are also affected by an rms fluctuation of $\sim 5 \%$, which we also take into account (see Appendix B).

\section{B. Intensity control}

The possibility of creating beams of equal size at several intensities is important for proof of principle measurements. In order to keep the same initial beam profile at injection in SIS18, the intensity was changed directly via UNILAC. However, the linac-synchrotron injection scheme based on the "multiturn injection" is unavoidably affected by the change of the horizontal tune $Q_{x}$. This drawback cannot be avoided and makes a difference with respect to the PS experiment [18], where the bunch to bucket transfer kept the intensity virtually independent of the machine tunes. The experimental results show that this effect can be estimated for this experiment to fluctuate as much as $\pm 15 \%$, which also includes the intensity fluctuation of the source.

\section{THE MEASUREMENT CAMPAIGN}

\section{A. Low intensity coasting beam}

The finding of the SIS18 resonances is the result of an experimental campaign performed in 2004 (see Refs. [25,26]). In Fig. 4 we show these results obtained by scanning the machine tunes across the working diagram and measuring beam loss (see scale for relative loss, blue signifies no loss). The choice of the lattice resonance for the high intensity trapping studies is based on Fig. 4. The best option for studying the long-term high intensity effects is given by the resonance $3 Q_{x}=13$ which allows enough surrounding space in the tune diagram to allocate some space charge tune spread without intercepting any other resonance. As pointed out in the Introduction, the simultaneous presence of chromatic effects and space charge creates a complex mixed dynamics, which in this experiment we prefer to avoid in order to focus on purely space charge driven incoherent effects. In fact, our coasting beam has $(\delta p / p)_{\mathrm{rms}} \sim 4.8 \times 10^{-4}$ which combined with a natural chromaticity of $\xi_{x}=\left[\partial Q_{x} / \partial(\delta p / p)\right] / Q_{x} \simeq-1.5$ gives an unwanted rms tune spread of $\pm 3 \times 10^{-3}$. Therefore

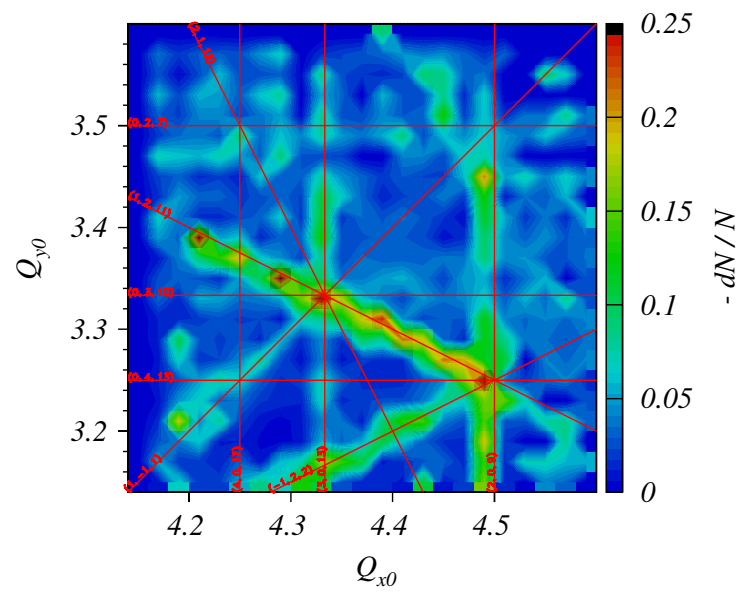

FIG. 4. Experimental resonance chart of SIS18. The colors represent the normalized beam loss between two consecutive data acquisitions $(10 \mathrm{~ms})$ during several tune scans each $1 \mathrm{~s}$ long [25]. In these measurements the chromaticity was left uncompensated.
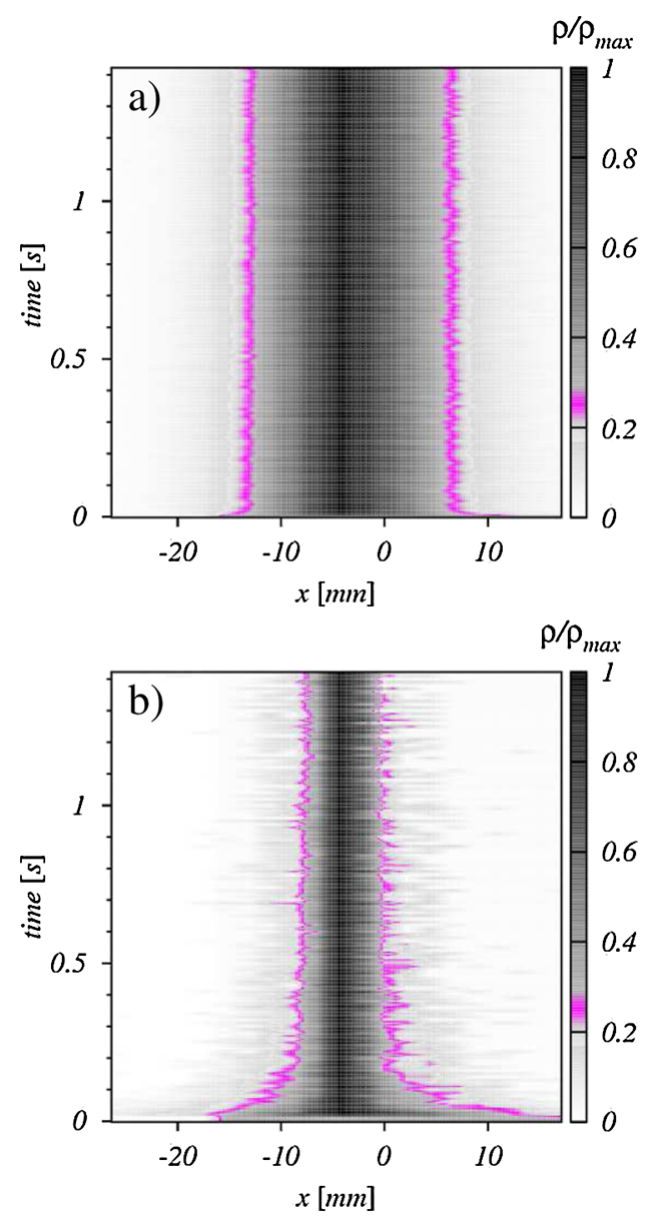

FIG. 5. Low intensity coasting beam: Time evolution of beam profile for (a) $Q_{x}=4.3185, Q_{y}=3.245$; (b) $Q_{x}=4.3325$, $Q_{y}=3.245$. 
we required that in all measurements the chromaticity is always to the best of our ability compensated, and as the resonances measured in Fig. 4 were obtained with uncompensated chromaticity, the effective stop band of the resonance $3 Q_{x}=13$ was measured again. In order to reach this goal, we have measured a coasting beam response in terms of emittance growth and beam loss for several horizontal tunes keeping $Q_{y}=3.245$. In these measurements the intensity is kept low so as to resolve the pure single particle nonlinear effects by injecting $\sim 0.8 \times 10^{9}$ ions. For each working point we have stored the transverse profiles to reconstruct the time evolution of the beam properties as shown, for the example of a tune off the 3 rd order resonance at $Q_{x}=4.3185$ in Fig. 5(a), and for a tune in the beam loss stop band at $Q_{x}=4.3325$ in Fig. 5(b). In both these pictures and all those of the same type throughout this paper, the red-pink region provides a reference for the beam edge taken at $25 \%$ of the beam peak intensity. In Fig. 5(b) the beam edge shows the dramatic effect of the 3rd order resonance: In the first $100 \mathrm{~ms}$ of beam storage a substantial beam loss takes place at the expense of the beam size.
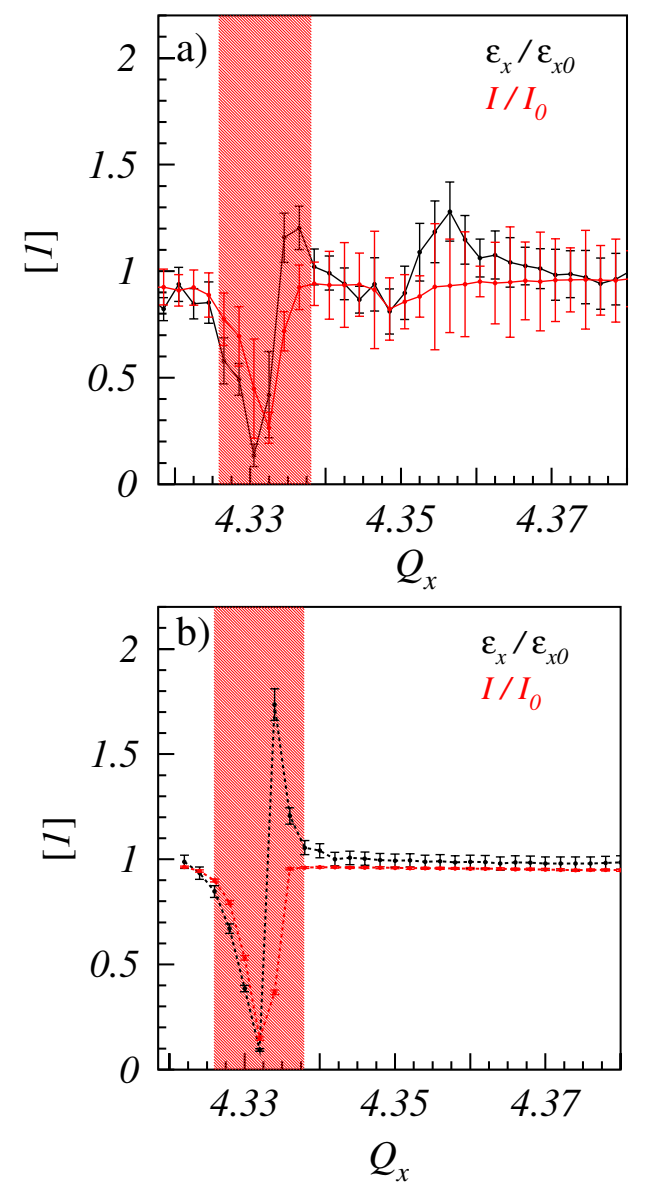

FIG. 6. Low intensity coasting beam: (a) Measured normalized emittance increase and beam loss versus $Q_{x}$. (b) Simulation of beam loss and emittance increase: The stop band of beam loss allows the calibration of the strength of the nonlinearities in the lattice model.
Afterwards the beam size stabilizes as a result of the complete scraping of all particles out of the 3rd order separatrix. The figure of merit of what happens to the beam is obtained defining an emittance ratio $\epsilon_{x 1} / \epsilon_{x 0}$, where $\epsilon_{x 0}$ is the emittance of the beam at injection and $\epsilon_{x 1}$ is the beam emittance after $1 \mathrm{~s}$ storage. The summary of all these measurements is shown in Figs. 6(a) where a beam loss stop band of $\Delta Q_{x} \simeq 0.012$ due to the 3 rd order resonance $3 Q_{x 0}=13$ is found. The region of the 3 rd order resonance induced beam loss is marked by a red stripe as reference for other measurements, which will be presented in this paper. The red and the black curves show, respectively, the beam survival and the emittance ratio after $1 \mathrm{~s}$ storage. The error bar shown in Figs. 6(a) and 6(b) and all other pictures throughout this paper are discussed in Appendix B.

\section{Modeling the nonlinear dynamics in the proximity of the 3 rd order resonance}

The measurement of the SIS18 nonlinear components is difficult and is part of an undergoing effort. In fact, the data acquisition of the beam position monitor (BPM) system does not allow for a simultaneous data acquisition from two BPMs. Therefore we cannot use a resonance driving term method [27], and we pursue the goal of measuring the nonlinear components with an alternative method based on deforming the closed orbit $[28,29]$. Hence, the known nonlinear optics of SIS18 includes only the six sextupoles for chromatic correction since the natural errors are not known. The presence of the six identical correction sextupoles alone cannot explain the experimental findings as they drive the 6th harmonics and therefore do not excite the resonance $3 Q_{x}=13$. As the locations of the sextupolar errors creating the 13th harmonics are not known, we make the ansatz of a nonlinear model of SIS18 with only one sextupolar error. It is shown in Appendix A that a Gaussian beam of rms emittance $\tilde{\epsilon}_{x}$ in a linear lattice equipped with one sextupole of integrated strength $K_{2}$, creates the beam loss stop band $\Delta Q_{x, \mathrm{sb}}$ (defined for a beam loss of $1 \%$ )

$$
\Delta Q_{x, \mathrm{sb}}=0.217 \sqrt{\tilde{\epsilon}_{x}} \beta_{x}^{3 / 2}\left|K_{2}\right|,
$$

where $\beta_{x}$ is the beta function at the location of this error sextupole. We therefore add an error sextupole located in proximity of a dipole, where $\beta_{x}=5 \mathrm{~m}$, and apply Eq. (1) to a beam of $\epsilon_{x, \text { rms }}=4.75 \mathrm{~mm}$ mrad requiring a beam loss stop band of $\Delta Q_{x, \mathrm{sb}} \simeq 0.012$ as observed in the measurements [Fig. 6(a)]. The integrated strength of this error sextupole is then set to $K_{2}=-2.2 \mathrm{~m}^{-2}$.

The discussion in Appendix A for deriving Eq. (1) shows that the pattern of beam loss created by one sextupole is symmetric in $Q_{x}-13 / 3$ [see Figs. 21(c) and 21(d)]. However, in Fig. 6(a) the measured beam loss (red curve) does not exhibit this symmetry, indicating the presence of an extra detuning acting on resonant particles. Clearly, the presence of relatively strong chromatic correction sextupoles contributes as a source of detuning, and the direction 
in which the detuning acts is essential for determining the direction of the beam loss asymmetry. Thus, it becomes necessary to take the sign of the error sextupole strength $K_{2}$ to be negative. Note that this procedure fixes $K_{2}$ and its sign, however, we found that this setting creates a too strong amplitude dependent detuning: Simulations have shown that the asymmetry in the beam loss pattern is too
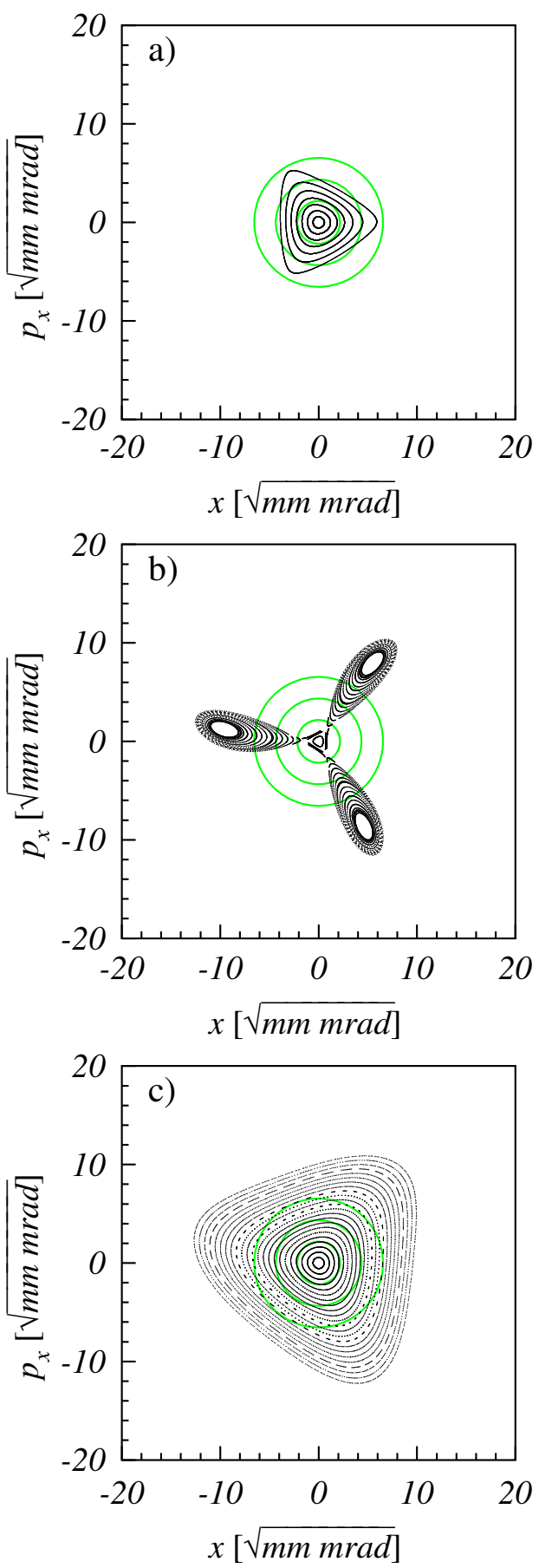

FIG. 7. Phase space portrait of the stable orbits for (a) $Q_{x}=4.328$, (b) $Q_{x}=4.334$, and (c) $Q_{x}=4.34$. The dynamics is determined only by the magnet nonlinearities. No space charge is included. strong with respect to that measured in Fig. 6(a). As the strength of the chromatic correction and error sextupoles are not free parameters, we counteract to the excessive amplitude dependent detuning by using an octupole. We find that a value $K_{3}=25 \mathrm{~m}^{-3}$ of the octupole strength fitted well the asymmetry of the beam loss in Fig. 6(a).

This integrated nonlinear component can be attributed partly to the pseudo-octupoles created by the quadrupole fringe field [30,31]. The focusing quadrupoles are each $1 \mathrm{~m}$ long with a strength of $K_{F}=0.31 \mathrm{~m}^{-2}$. By taking the approximations of Ref. [32] we find the strength of the pseudo-octupoles to be $K_{3, \text { po }}= \pm 3.6 \mathrm{~m}^{-3}$. For the defocusing quadrupoles we find $K_{3, \mathrm{po}}= \pm 5.88 \mathrm{~m}^{-3}$. The strength of these local kicks is smaller by a factor $\sim 5-8$ than the single octupole used in the modeling. Therefore these nonlinear kicks distributed over 24 quadrupoles (with alternated signs) are not the source of the large octupolar strength assumed in our model.

The effect of all the nonlinearities included in this SIS18 model are visualized in the Poincaré sections shown in Fig. 7. We plot three cases: Fig. 7(a) shows a phase space plot in Courant-Snyder (CS) coordinates for a working point below the resonance at $Q_{x}=4.328$. The characteristic triangular shape of the stable orbits is obtained. Note that the stable fixed points are located at infinity in the absence of other source of nonlinearities. As a reference we draw three circles in correspondence of $1 \sigma_{x}, 2 \sigma_{x}$, and $3 \sigma_{x}$ of the beam distribution. From this picture it is visible that a fraction of the beam lies outside of the separatrix, and will therefore become lost. This is visible in Fig. 6(b) and a small beam loss is also seen in the measurement in Fig. 6(a). The effect of the nonlinearities driving the resonance is shown in Fig. 7(b) for the working point $Q_{x}=4.334$. Note the small triangular separatrix and three new stable islands with fixed points located at positions far from the beam edge. These new islands appear as a consequence of the nonlinear detuning created by the chromatic correction sextupoles and the extra octupole used to match the model to the experimental beam loss. At this working point there is a large particle loss, but also some of the particles remain bounded in their motion in the new stable islands, creating an rms emittance increase [as seen in Fig. 6(b)]. The same effect is seen in Fig. 6(a).

In Fig. 7(c) the working point $Q_{x}=4.34$ located above the resonance is used. A vertical mirroring of the stability region takes place because of the change of sign of the distance of the bare tune from the resonance. The rounding of the triangular shape indicates that this tune is away from the third order resonance. Nothing dramatic is expected to happen to emittance growth and beam loss ratio as is evident from Figs. 6(a) and 6(b).

\section{Multiparticle simulations}

By constructing a computer model taking functionality from the MICROMAP library [33], the effect of the lattice 
with its nonlinearities on the stored beam could be simulated. Thus, the normalized beam loss and emittance after $1 \mathrm{~s}$ of storage time for the same working points as used in the measurements were computed. In the simulations we used 2000 macroparticles which are removed when they exceed the machine acceptance. The space charge is computed with a frozen model described in Ref. [34]. For all investigations, the lattice was maintained with a chromaticity which was compensated by a family of sextuples.

The results of the simulations in Fig. 6(b) show an acceptable approximation to those measured in Fig. 6(a). It is evident that the SIS18 nonlinear model does not reproduce the peak in the emittance growth found at $Q_{x} \simeq$ 4.356 as it does not include the driving term of the underlying resonance. The modeling of this unknown resonance is not possible with the information available at present. In Fig. 6(a) the measured emittance growth at $Q_{x}=4.3365$ exhibits an increase of $20 \%$ above the resonance (peak in the stop band). This is interpreted again as an effect being caused by amplitude dependent detuning which pushes particles out of the stop band before being lost. The resulting emittance increase depends on the details of the synchrotron, but especially on the trade-off between the strength of the amplitude dependent detuning and free space left in the beam pipe for the beam to blow up (or ratio of the acceptance to beam emittance). In our simulations in Fig. 6(b), we observe a similar effect. The peak in the emittance increase is an unavoidable consequence of neglecting natural nonlinearities of SIS18.

We will use this nonlinear model for the simulations of the high intensity beam dynamics of SIS18 throughout this paper. Given the high CPU time demand of these simulations, we limit our study to the experiment settings avoiding additional parameter scan studies.

\section{Calibration of the SIS18 tunes}

In SIS18 the tunes are inputs to the accelerator control system, but the actual tunes experienced by a beam particle differ from these set-point values. In fact, the resulting tune depends on the "feed down" of nonlinear components due to closed orbit deformations in addition to an intrinsic calibration shift present in the SIS18 control system. As in this paper we will compare experimental measurements with simulations, we remove this ambiguity by calibrating the tunes given to SIS18 with the simulation model. In fact, when beam loss is maximum and asymptotic beam size small, the main resonant process comes from the third order resonance without additional contributions from other effects. The SIS18 tune corresponding to this situation is found at $\tilde{Q}_{x}=4.2960$, which cannot be taken as the center of the 3rd order resonance. We correct this tune by adding a shift of $\Delta Q_{x}=0.0365$, to overlap the maximum beam loss of the measurements [Fig. 6(a)] with the tune corresponding to the maximum beam loss found in the simulations [Fig. 6(b)]. This is the only correction applied to all the measured data throughout this paper. The error bar assigned to the tunes is $6 \times 10^{-4}$ as discussed in Appendix B.

\section{B. High intensity coasting beam}

The experimental study of the high intensity effects on the single particle nonlinear dynamics, i.e. trapping or scattering of particles on stable islands, can be done mainly by measuring global beam quantities, such as transverse and longitudinal beam size and beam loss. However, these observables can also vary because of the presence of other effects invalidating the purpose of the measurements. We therefore perform a preliminary study in order to establish whether significant variations of these observables are found. As a first step we studied the beam response in a high intensity coasting beam.

We injected into SIS18 $3 \times 10^{9}$ ions, which create a peak incoherent space charge tune shift of $\Delta Q_{x, \mathrm{sc}}=$ $-0.025, \Delta Q_{y, \mathrm{sc}}=-0.03$. These values have been obtained for a Gaussian beam distribution with the formulas [35]

$$
\begin{aligned}
& \Delta Q_{x, \mathrm{sc}}=\frac{\bar{\beta}_{x}}{2} R_{\mathrm{acc}} \frac{K_{\text {peak }}}{\sqrt{\bar{\beta}_{x} \epsilon_{x}}\left(\sqrt{\bar{\beta}_{x} \epsilon_{x}}+\sqrt{\bar{\beta}_{y} \epsilon_{y}}\right)} \\
& \Delta Q_{y, \mathrm{sc}}=\frac{\bar{\beta}_{y}}{2} R_{\mathrm{acc}} \frac{K_{\text {peak }}}{\sqrt{\bar{\beta}_{y} \epsilon_{y}}\left(\sqrt{\bar{\beta}_{x} \epsilon_{x}}+\sqrt{\bar{\beta}_{y} \epsilon_{y}}\right)},
\end{aligned}
$$

where $\bar{\beta}_{x}=R_{\text {acc }} / Q_{x 0}, \bar{\beta}_{y}=R_{\text {acc }} / Q_{y 0}$ are the average horizontal/vertical beta functions, respectively, and $R_{\text {acc }}$ is the accelerator radius. These formulas are valid for small tune shift and long bunches, i.e. $r_{x} / r_{z} \ll 1$, which is the case in our experimental condition. The factor $K_{\text {peak }}$ is the peak perveance given by $K_{\text {peak }}=q I_{\text {peak }} /\left(2 \pi \epsilon_{0} m c^{3} \beta^{3} \gamma^{3}\right)$, where $q$ is the charge of the ion, $I_{\text {peak }}$ is the peak beam current, $\epsilon_{0}$ the vacuum permittivity, $m$ is the ion mass, and $\beta, \gamma$ are the relativistic factors. This formula, due to the optics, underestimate shifts in the horizontal tune of $20 \%$, and in the vertical tune of $2 \%$. Note that the $I_{\text {peak }}$ for a coasting beam is obtained from the average intensity.

The high intensity current was chosen with the strategy of creating "equivalent beams." Ideally we require that coasting beams at high intensity have the same space charge tune spread of high intensity bunched beams (Sec. III D) so we can compare the beam dynamics under the same space charge condition for phenomena of periodic resonance crossing. Clearly, this must take the bunching factor into account. This allowed us to determine an intensity for the bunched beam of $\sim 1.2 \times 10^{9}$ ions and the closer "equivalent" intensity for the high intensity coasting beam reached in the experiment is of $\sim 3 \times 10^{9}$ ions. The low intensity measurements should be instead with zero intensity, but we have been forced to keep a minimum beam intensity in order to avoid a too strong noise to signal measurement condition. Again we keep the beam intensity for the low intensity bunched beam lower than that for the 
low intensity coasting beam (see Table II for a summary of beam intensities).

In Fig. 8(a) we show the results of these measurements. This picture shows explicitly the detuning effect induced by space charge: The maximum beam loss at $Q_{x}=4.3365$ reduces the beam survival to $72 \%$. This minimum is found shifted to the right of that in Fig. 6(a), where it is located at $Q_{x}=4.3325$ and $I / I_{0}=0.26$. A similar pattern is found for the peak value of the emittance ratio 1.29 which occurs at $Q_{x}=4.3425$, whereas in Fig. 6(a) it is located at $Q_{x}=$ 4.3365 with $\epsilon_{x, 1} / \epsilon_{x, 0}=1.2$. The peak in emittance growth is shifted by $\Delta Q_{x, s} \simeq 0.006$. We do not expect that this shift derives from a coherent resonance effect [36-38]. In fact, in Fig. 8(b) the simulations are obtained using a frozen model of the space charge force created by the beam [34], which does not incorporate coherent effects. Therefore the shift in the emittance growth curve is due to the incoherent space charge effect, while the coherent character of the interplay of the space charge with the 3rd order resonance would be found closer to the stop band (at $Q_{x}=4.3365$ )
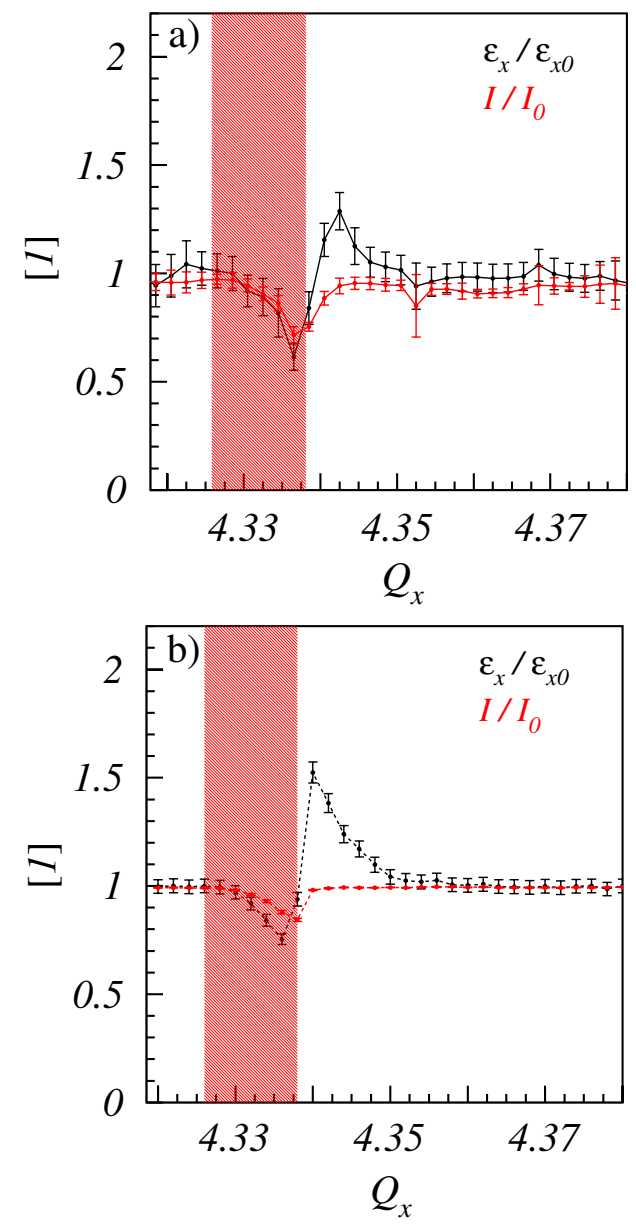

FIG. 8. High intensity coasting beam: (a) Normalized emittance increase and beam loss versus $Q_{x}$. (b) Simulation of beam loss and emittance increase versus $Q_{x}$. For this simulation the beam intensity has been taken so as to create a maximum tune shift of $\Delta Q_{x} \simeq-0.025$. where the beam loss becomes substantial. In Fig. 8(b) the simulations of this measurement reproduce with acceptable accuracy the experimental results. The peak in the emittance increase is again shifted by $\Delta Q_{x, s} \simeq 0.0075$. Note the amount of beam loss, which is less than in the measurement. This discrepancy may be attributed to the lack of self-consistency in simulations. It is interesting that both simulations and measurements show that the presence of space charge mitigates beam loss. The space charge detuning plays a beneficial role in bringing particles out of the 3rd order resonance. Only the particles which are at large amplitudes cannot receive this effect and therefore are lost in the resonance stop band. It is also remarkable that any trace of the second stop band found in Fig. 6(a) disappears. The explanation for this finding is beyond the purpose of this paper as we do not possess enough information on all resonances of the SIS18.

In order to visualize the effect of the space charge, we plot in Fig. 9 the phase space portraits of the same working points used in Fig. 7. Comparing Fig. 9(a) with Fig. 7(a) we note immediately the effect of the detuning which enlarges the stability region. Even more dramatic is this effect for the working point $Q_{x}=4.334$ [Fig. 9(b)] if compared with Fig. 7(b); the stable region becomes very large in contrast to the very small triangular domain of Fig. 7(b). In Fig. 9(c) the space charge brings the 3rd order resonance in an otherwise stable region of the phase space. The position of the three stable islands is controlled by the strong space charge detuning and the machine working point. These islands, in spite of being in a stationary position, play a role in the increasing of emittance.

In fact, part of the beam tails overlap with the islands and are therefore subjected to the dynamics of the resonance creating an emittance increase seen in Fig. 8(b) at $Q_{x}=$ 4.34. For larger working points, these stable islands are located closer to the transverse origin creating a smaller emittance increase [see in Fig. 8(b)] as their amplitude decreases and the fixed points are closer to the origin. The same argument is a possible explanation for the experimental findings of Fig. 8(a). In order to show this clearer, we plot in Fig. 10 as a function of the working point $Q_{x}$, the further and closer distance of the separatrix from the origin. The island edges are rescaled with respect to the rms beam size. It is evident for $Q_{x}>4.35$ that the outer edge of the island is located inside the beam, hence causing a small or negligible emittance increase. In terms of tune footprint, the comparison of the effect of the lattice nonlinearities with respect to the case in which space charge is added is also useful. Each tune-footprint picture is obtained from a series of test particles placed in the area $-5.15 \sigma_{x}<x<5.15 \sigma_{x}$, and $-5.83 \sigma_{y}<y<5.83 \sigma_{y}$, i.e. $-4 \mathrm{~cm}<x, y<4 \mathrm{~cm}$ on a grid of $401^{2}$ points. All the other coordinates are taken zero (hence all particles are taken to be at $z=0$ in the bunch frame). In Fig. 11 on the left are shown the tune footprints driven by the lattice 

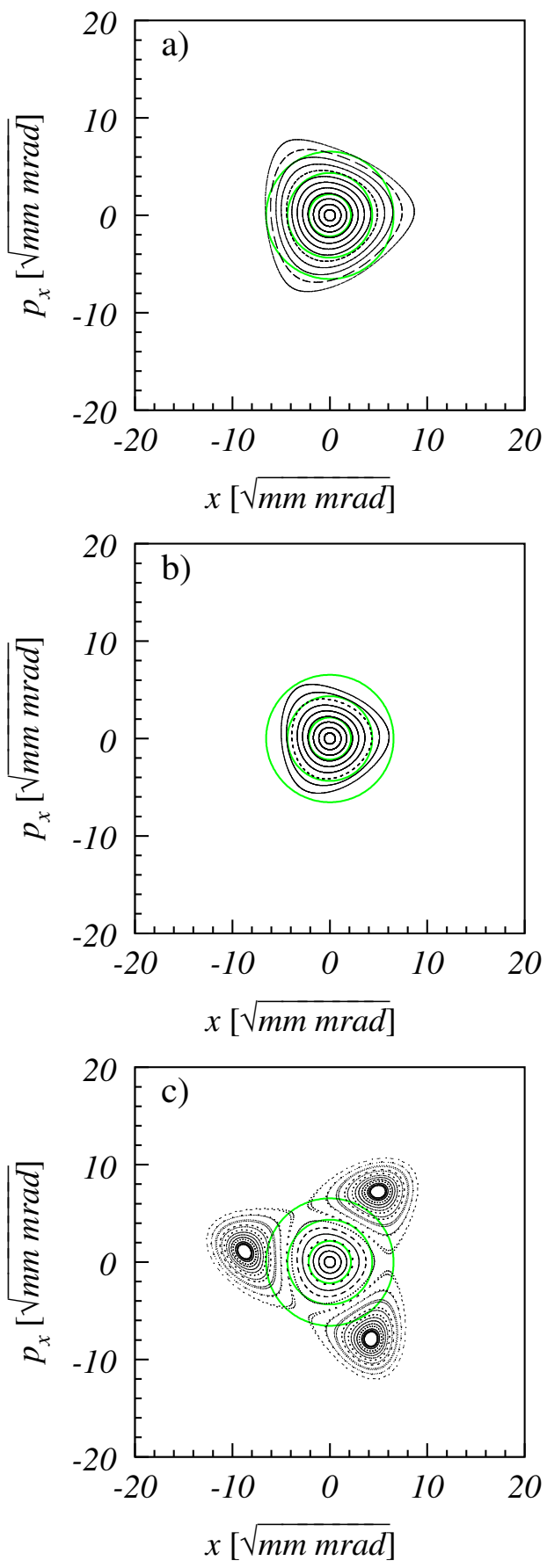

FIG. 9. Phase space portrait of the stable orbits for (a) $Q_{x}=4.328$, (b) $Q_{x}=4.334$, and (c) $Q_{x}=4.34$. In the particle dynamics are included, the magnet nonlinearities and the beam space charge.

nonlinearity. The detuning is minimum for particles at small transverse amplitudes which are located very close to the bare tune. For particles at large amplitudes the detuning is positive and in Fig. 11(c) it is visible as a vertical line in correspondence to the resonance $3 Q_{x}=$ 13. The particles with tunes locked on this line are those that are on the three islands in Fig. 7(b). In the pictures on the right column of Fig. 11 we plot the tune footprints in

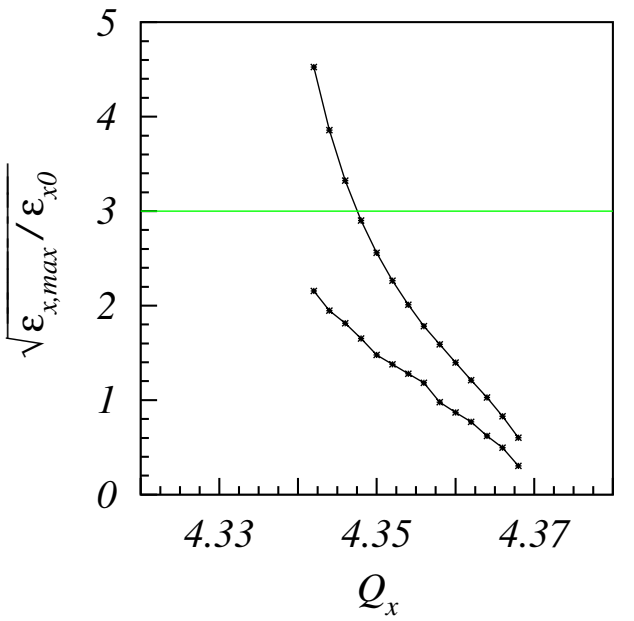

FIG. 10. Position of the 3rd order island edges in units of rms beam size as a function of the working point $Q_{x}$. The green line is located at the edge of the beam.
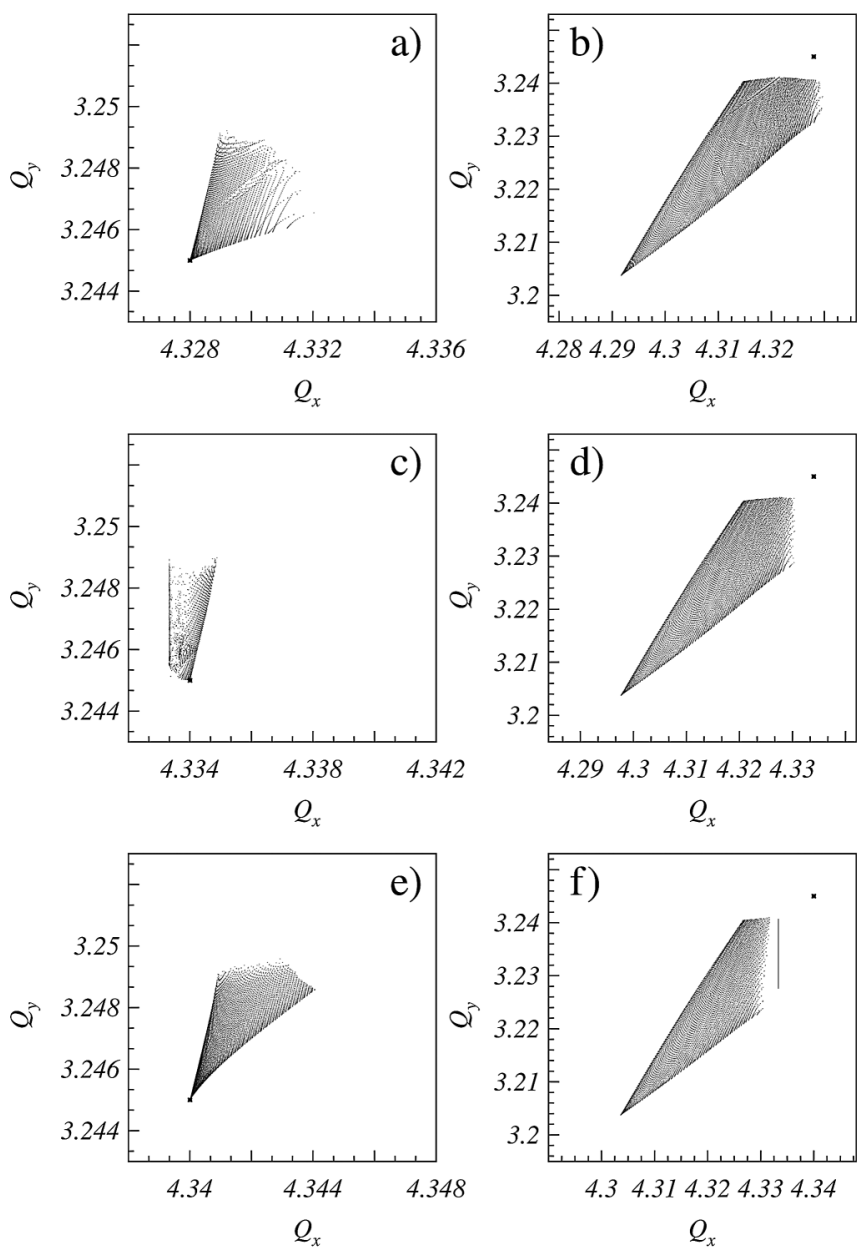

FIG. 11. Tune footprints: In pictures (a), (b) the tune is $Q_{x}=$ 4.328 , in (c), (d) the tune is $Q_{x}=4.334$, in (e), (f) the tune is $Q_{x}=4.34$. The pictures on the left provide the tune footprint from the pure nonlinear lattice. The pictures on the right include also the effect of the space charge. Note that space charge has a dominant effect over the detuning of the lattice nonlinearities. 
the presence of space charge and lattice nonlinearities for the reference intensity of $N=2.5 \times 10^{8}$ ions per bunch. The negative detuning of the space charge overcomes the positive detuning of the lattice nonlinearities. This dramatic effect is visible in Fig. 11(d), where all particles are brought away from the resonance or are otherwise brought into the resonance as is the case in Fig. 11(f). The resonant particles of Fig. 11(f) are those populating the three islands of Fig. 9(c).

\section{Low intensity bunched beam}

The study of high intensity effects on a bunched beam requires the control of the off-energy related effects as well as the creation of a well controlled stable bunched beam. Stable bunches have been created by means of a special $\mathrm{rf}$ cycle which is applied in addition to the standard SIS18 rf cycle; $100 \mathrm{~ms}$ after the beam is injected the coasting beam is adiabatically bunched in $10 \mathrm{~ms}$ corresponding to 14 (linear) synchrotron periods. Afterwards the bunched beam is stored for $1 \mathrm{~s}$ ( $\mathrm{rf}$ parameters in Table I). The resulting linear synchrotron tune $Q_{s}$ yields one synchrotron oscillation in 144 turns. Longitudinal tune and the expected rms momentum spread are reported in Table I. After the storage is ended the beam is debunched in $100 \mathrm{~ms}$ and afterwards the standard SIS18 rf cycle takes over forming again a bunch, which is then accelerated and extracted at an energy of $400 \mathrm{MeV} / \mathrm{u}$. The presence of uncompensated chromaticity during the storage of a bunched beam is, for our purposes, unacceptable. In the presence of a natural normalized chromaticity of $\xi_{x} \simeq-1.5$ an rms tune spread of $\Delta Q_{x, \text { rms }}^{\mathrm{ch}}= \pm 8.3 \times 10^{-3}$ would be found, and a particle with off momentum $\delta p / p$ would be subjected to a tune variation function of the turns $n$ as $\delta Q_{x}^{\text {ch }}=Q_{x} \xi_{x}(\delta p / p) \times$ $\cos \left(2 \pi Q_{s} n\right)$. This effect interferes with the dynamics of resonance crossing induced by space charge. For this reason in our measurements the chromaticity is compensated via the SIS18 control system. However, no further measurements were performed to control the effectiveness of the chromatic correction and the presence of some residual chromaticity should not be excluded.

In order to distinguish the relevance of this type of effect from the pure space charge driven beam blowup we perform measurements, with weak space charge, to assess the effect arising from the pure residual chromaticity and by the presence of the dispersion. For this measurement the SIS18 was filled with $\sim 0.4 \times 10^{9}$ ions. Figure 12 shows the horizontal beam evolution for two different horizontal tunes. Note in Fig. 12(a), obtained at $Q_{x}=4.3185$, the small beam size increase attributed to the larger momentum spread following bunch formation. At the end of the storage time when the debunching takes place (in $100 \mathrm{~ms}$, i.e., 140 synchrotron periods), the extra beam size is removed because the debunching processes are to a good approximation adiabatic. The same process is better visible, because of the higher intensity, in Fig. 15(a). If $\rho(x)$ is
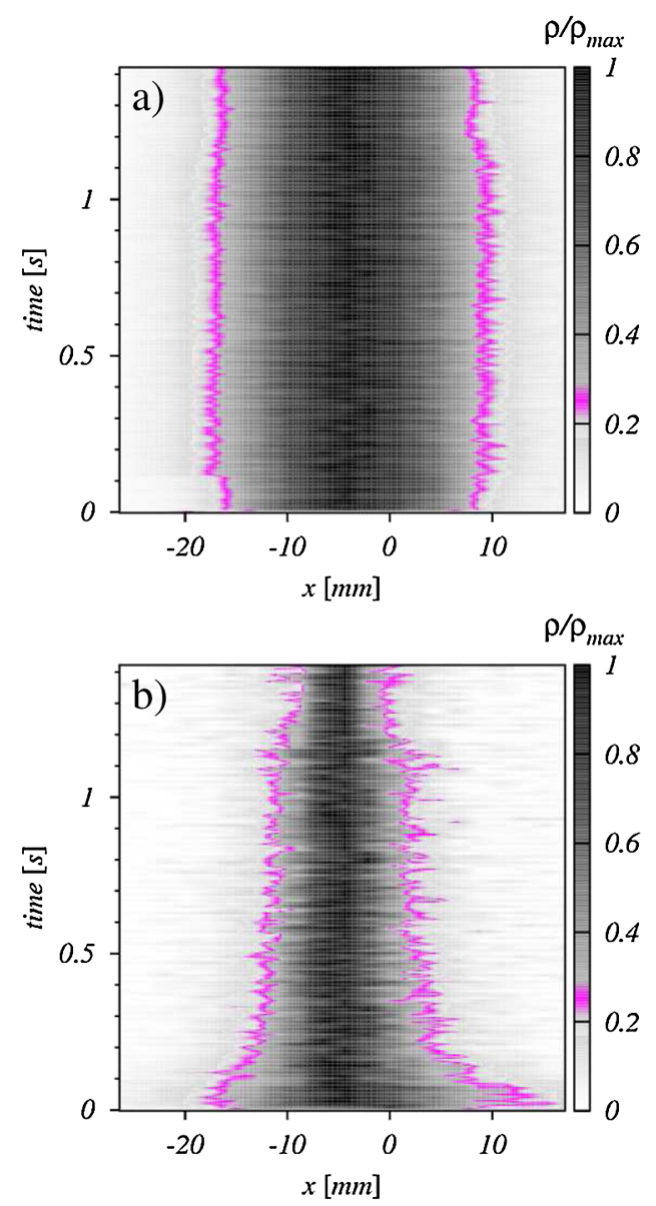

FIG. 12. Low intensity bunched beam: (a) Beam evolution at $Q_{x}=4.3185$. (b) Beam evolution at $Q_{x}=4.3325$.

the horizontal beam profile in the absence of momentum spread, and the beam size $x$ is measured at $\rho(x) / \rho_{\max }=\alpha$ [the red edge in Fig. 12(a)], then a momentum spread of $(\delta p / p)_{\text {rms }}$ changes the beam edge according to

$$
\frac{\Delta x}{x} \simeq-\ln (\alpha)\left[\frac{D_{x}(\delta p / p)_{\mathrm{rms}}}{x}\right]^{2} .
$$

Because the dispersion at the position of the IPM is $D_{x}=$ $1.6 \mathrm{~m}, D_{x}^{\prime}=0.2 \mathrm{mrad}$, and $\alpha=0.25$, we find $\Delta x / x \simeq 4 \%$, i.e. an increase of beam edge of $\Delta x \sim 0.5 \mathrm{~mm}$. Figure 12(a) shows instead a beam size increase of $\simeq 1.5 \mathrm{~mm}$. The discrepancy of the measurement with the predictions of Eq. (3) is attributed to the discretization of the beam profile measurement; in fact the anode wires present in the multichannel plate of the IPM have a pitch of $2.1 \mathrm{~mm}$ (thickness of the wires $1.5 \mathrm{~mm}$ and distances between wires $0.6 \mathrm{~mm}$ ), which sets the effective resolution of the device for the beam edge measurements. Figure 12(b) shows the horizontal beam evolution in the resonance stop band at $Q_{x}=$ 4.3325. The effect of the resonance is fully visible. In the first $10 \mathrm{~ms}$ the increase of the beam profile is possible because of a small beam loss rate. After $0.2 \mathrm{~s}$ the large beam loss almost saturates while the beam size slowly decreases, keeping a larger size than in Fig. 5(b). 
An adequate assessment of the long-term emittance growth should be clearly done by measuring beam profiles before/after the adiabatic bunching/debunching, respectively, thereby avoiding artifacts due to the dispersion and the increased $\delta p / p$. The summary of the beam response for this sequence of measurements is shown in Fig. 13(a). In this picture we find that the peak emittance $\epsilon_{x, 1} / \epsilon_{x, 0}=1.22$ on the right of the 3rd order stop band at $Q_{x}=4.3385$ has still an unchanged amplitude with respect to the value found in Fig. 6(a) $\left(\epsilon_{x, 1} / \epsilon_{x, 0}=1.20\right)$ and Fig. 8(a) $\left(\epsilon_{x, 1} / \epsilon_{x, 0}=1.29\right)$.

The peak beam loss, $I / I_{0}=0.31$ at $Q_{x}=4.3325$, practically remains as in Fig. 6(a) although the beam loss stop band has slightly widened. This result can be attributed to a residual chromaticity still present after chromaticity compensation. In Fig. 13(b) we show the simulation results for the modeling of these measurements. We find that simulations of the beam loss predict well the experimental findings within the stop band. The large emittance growth on the right of the resonance does not exceed the same peak found in the simulation of Fig. 6(b).
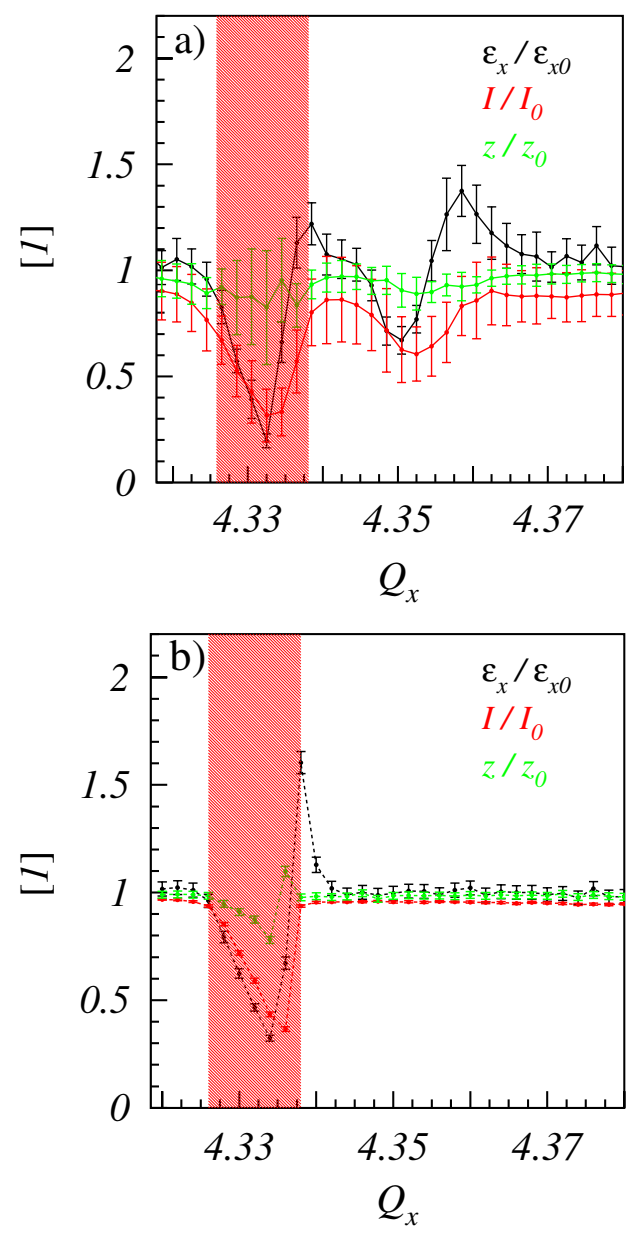

FIG. 13. Low intensity bunched beam: (a) Normalized emittance increase, bunch length, and beam loss versus $Q_{x}$. (b) Simulation of beam loss, bunch length, and emittance increase.
From these measurements we conclude that the residual chromaticity in this experiment is not significant enough to introduce extra effects in the periodic resonance crossing. The stop band is slightly enlarged, but the emittance increase is not affected. In Fig. 13(a) we present in addition the rms bunch length ratio as a function of the working point (green curve). The longitudinal profile measurements were recorded by a LeCroy oscilloscope ("WaveRunner 6000A") for digitizing and storing the BPM data.

For every tune about 4000 bunch profiles were recorded along the storage of $1 \mathrm{~s}$. The calculation of the bunch rms size at a given time $t$ is made by averaging the longitudinal profiles of the 30 neighboring profiles. Given the large number of measured profiles in $1 \mathrm{~s}, 30$ consecutive profiles make up $1 \%$ of the total, which are not significantly affected by beam variation. A detailed discussion on the error bar assigned to the rms bunch length is included in Appendix B. The green curve in Fig. 13(a) shows that at the location of the $3 \mathrm{rd}$ order beam loss stop band the rms bunch length becomes shorter. This may be a result of a reduction of the dynamic aperture and of a small
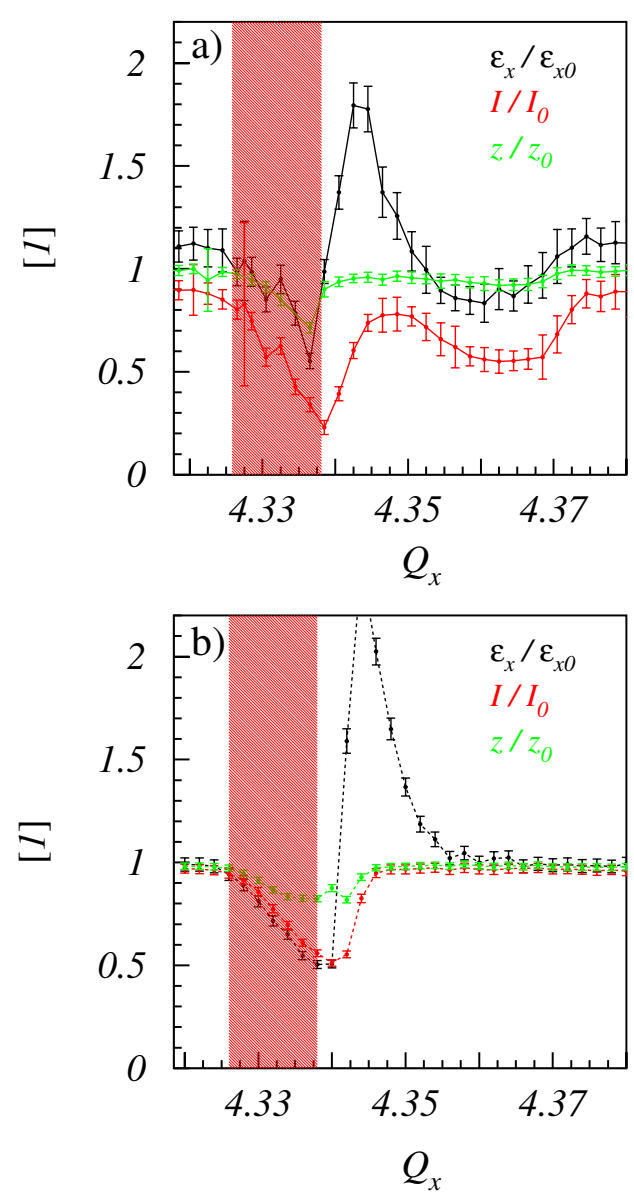

FIG. 14. High intensity bunched beam: (a) Measured transverse-longitudinal beam response to the long-term storage as a function of the working points around the third order resonance. (b) Simulation of the same case. 
enlargement of beam size due to the momentum spread: In fact, the phase space plots of Fig. 7 show that the region of stable motion decreases for tunes close the 3rd order resonance, (i.e. the dynamic aperture collapses on the resonance). At the same time, the beam size is slightly increased because of the $\delta p / p$ and residual chromaticity, hence particles with large $\delta p / p$ might be lost then perturbing the longitudinal beam profile. This feature is approximately observed in Fig. 13(b). Note the apparent bunch lengthening because particles are transported from the longitudinal core to the longitudinal tails of the beam.

\section{High intensity bunched beam}

All previous measurements had the purpose to assess: (1) the single particle 3rd order stop band (Sec. III A); (2) the effect of space charge on the coasting beam (Sec. III B); (3) the effect of the residual chromaticity on low intensity bunched beams (Sec. III C). We now present the study of the effect of high intensity on a bunched beam.

The high intensity bunches used in these measurements are characterized by a bunching factor and momentum
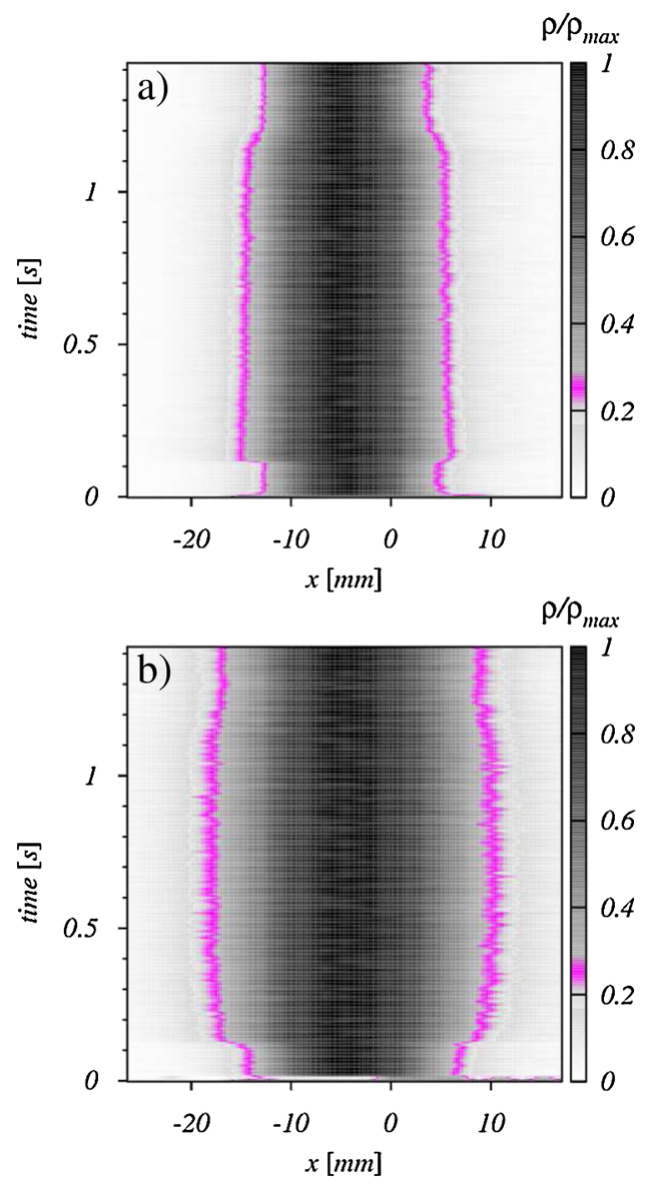

FIG. 15. High intensity bunched beam: (a) Waterfall $x$ beam evolution for $Q_{x}=4.3325, Q_{y}=3.245$. (b) Waterfall $x$ beam evolution for $Q_{x}=4.3425, Q_{y}=3.245$. spread as in Table I. The SIS18 was filled with $\sim 1.2 \times 10^{9}$ ions which yields an average peak space charge tune shift (directly measured from the IPM data) of $\Delta Q_{x, \mathrm{sc}} \simeq-0.04$, and $\Delta Q_{y, \mathrm{sc}} \simeq-0.045$. With this beam we repeat the same systematics around the 3rd order resonance as for the previous measurements in Sec. III C and collect the experimental findings in Fig. 14(a).

From this measurement it is evident that on the right of the 3rd order resonance, at $Q_{x}=4.3425$, in "absence" of beam loss, an emittance growth of $\epsilon_{x, 1} / \epsilon_{x, 0}=1.79$ is found while leaving the longitudinal bunch length unaffected. In Fig. 14(b) we show the simulation of this sequence of measurements. The curve of the beam loss has a pattern very close to the experimental value, but maximum beam loss is less than the measured value. The emittance increase exhibits instead a more pronounced growth than the measurements. We interpret this as a limit of our modeling of the nonlinear SIS18 lattice and lack of space charge self-consistency.

The effect of the high intensity on beam blowup is better seen by comparing the time evolution of beam profiles for certain relevant tunes. In Fig. 15(a) we show the beam evolution for the working point $Q_{x}=4.3325$, which previously exhibited the maximum beam loss [see Figs. 6(a) and 13(a)], but now has good beam survival because of the space charge detuning. Similarly, as for Fig. 12(a), the beam edge measured at $25 \%$ of the maximum beam profile intensity exhibits a pattern which reflects the adiabatic bunching/debunching processes. The beam edges are better resolved here than in Fig. 12(a) because of the higher intensity. In Fig. 15(b) we show the beam evolution for $Q_{x}=4.3425$ corresponding to the maximum emittance increase. The picture shows explicitly that after the debunching the beam edges do not return to their initial value. This effect is better shown in Fig. 16 where the horizontal

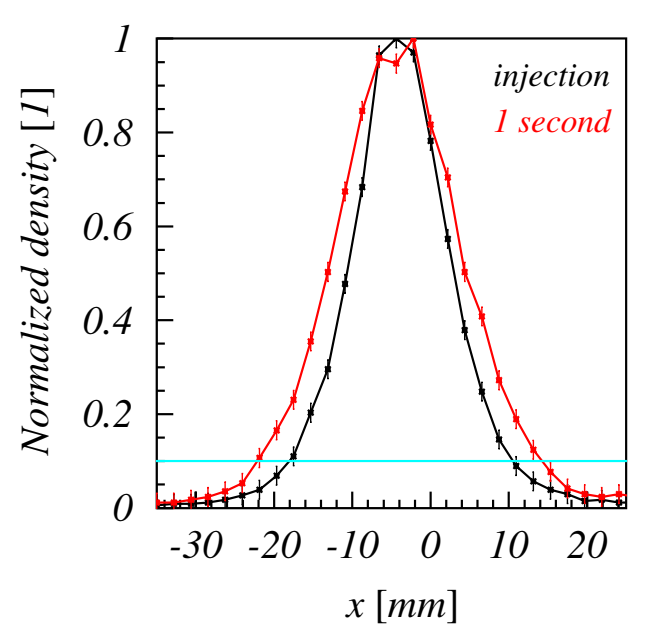

FIG. 16. High intensity bunched beam: Beam profile after injection and at the end of the storage time for $Q_{x}=4.3425$, $Q_{y}=3.245$. 

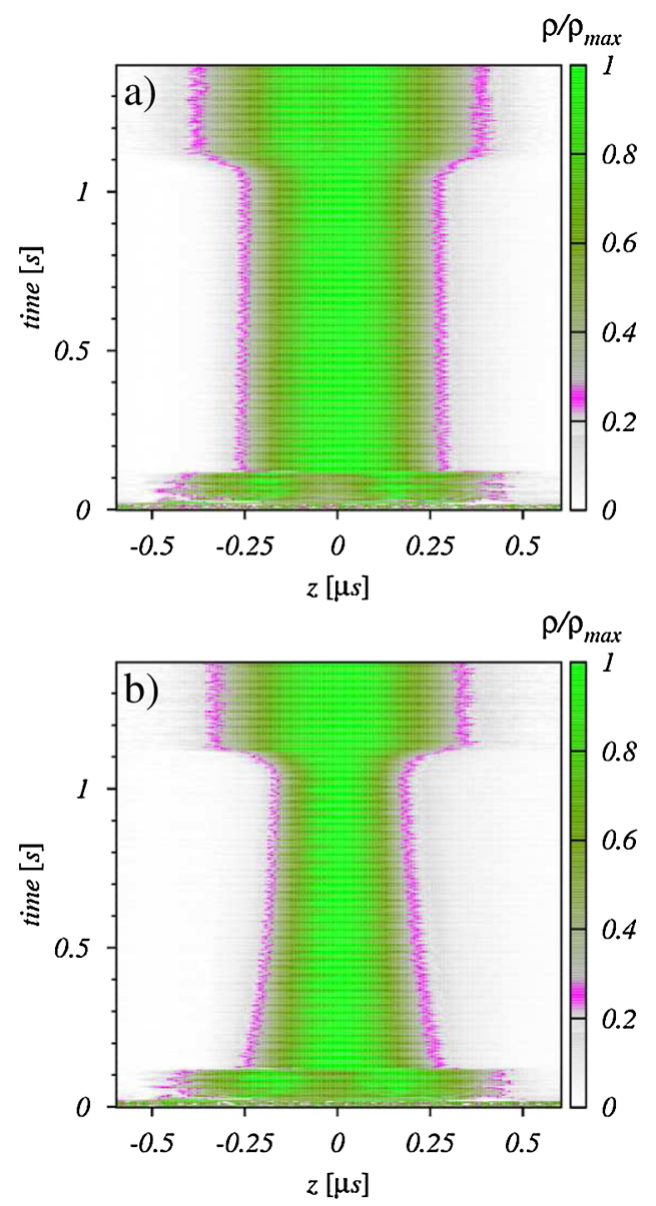

FIG. 17. High intensity bunched beam: Longitudinal beam evolution at $Q_{x}=4.3425$ and $Q_{x}=4.3365$. For the case $Q_{x}=$ 4.3365 the shortening of the beam tails is evident.

beam distribution before bunching and after debunching is compared.

The maximum beam loss is found at $Q_{x}=4.3385$ for a beam survival of $I / I_{0}=0.23$. For the measurement at a tune a step before, that is at $Q_{x}=4.3365$, a bunch shortening of $z / z_{0}=0.71$ is measured. The details of the longitudinal beam profile evolution are shown in Fig. 17 where in the picture on the top, for the tune $Q_{x}=4.3425$, i.e. relative to the maximum emittance growth, no relevant effects take place on bunch length. In Fig. 17(b) at $Q_{x}=$ 4.3365 we find that the edges of the bunch length shrinks in 0.9 s by up to $29 \%$.

\section{DISCUSSION}

In Fig. 14(a) we find a region of beam loss located above the resonance. At $Q_{x} \sim 4.342$ small beam loss and an emittance growth is observed. This distinction between a loss and an emittance growth regime was already observed in the CERN PS experiment. While trapping was suggested already in Ref. [18] as a possible explanation, we find that the present findings offer a firm ground to discuss the periodic resonance crossing due to the correlation between "coasting" and "bunched" as well as "low" and "high" intensity. This correlation is summarized in Table II.

The low intensity beams (bunched or coasting) exhibit the same emittance growth and similar maximum beam loss. Moreover, the tune at which the maximum beam loss or the maximum emittance increase is found is not significantly affected by the presence of the synchrotron motion. These findings show that no relevant effects due to the residual chromaticity are affecting the beam emittance growth and maximum beam loss.

The case for the high intensity beams is different: The maximum emittance growth is shifted to the right of the third order resonance. The beam loss behaves differently for the coasting beam with respect to the bunched beam. The maximum beam loss for the coasting beam is much less than for the bunched beam. The higher beam survival of the coasting beam stems from the detuning induced by space charge. The maximum emittance growth for coasting beams is almost the same at high intensity as at low intensity. Only when space charge and synchrotron motion are simultaneously present a large emittance growth is found. Although the maximum tune spread is not the same for bunched and coasting beam, we conclude that the experimental findings confirm that the emittance growth found in Fig. 14(a) is the result of the combined effect of high space charge and synchrotron motion, which induces a periodic resonance crossing. For completeness we report in Table III the comparison of the experimental findings with simulations. From this table we find that the ratio between the simulated and experimental maximum emittance growth lies in the range [1.32, 1.45]: The high intensity coasting beam has a maximum emittance growth which is nearly the same in both experiment and simulations. Therefore we conclude that the simulations are consistent with the experimental findings and the differences in the maximum emittance growth are somewhat due to the results of the incomplete modeling of the nonlinear SIS18 lattice.

The comparison of the experimental long-term beam loss with those predicted by simulations is more difficult. The long-term beam survival differs of a factor 2 between experiment and simulations in the case of the high intensity bunched beam, while for the coasting beam low intensity the survival in the simulations is half of that found in measurements. A possible source of this discrepancy is in modeling of the machine acceptance, made also difficult by the presence of a distorted closed orbit $(\sim 2-3 \mathrm{~mm})$. These uncertainties to the experimental conditions and the complexity of the dynamics make the long-term emittance growth and beam loss prediction difficult to be simulated with high accuracy.

Another finding of the PS experiment was that for a high intensity bunched beam, beam loss is accompanied by 
TABLE II. In this table are summarized the values of the essential quantities measured in each of the four measurement sets: Space charge strength, maximum beam loss, maximum emittance increase, and bunch length at the end of the storage. These quantities are grouped in rows according to the intensity of the beam (low/high intensity) and in columns according to whether the beam is bunched or coast. The column $Q_{x}$ provides the horizontal tune at which those quantities are measured. In all measurements the vertical tune has been kept constant at $Q_{y 0}=3.245$. From this table it is shown that only when high intensity and synchrotron motion are simultaneously present large emittance growth occurs as a result of the space charge induced periodic crossing of the third order resonance. The uncertainties in these parameters are the same as those in Figs. 6, 8, 13, and 14.

\begin{tabular}{|c|c|c|c|c|}
\hline & \multicolumn{2}{|c|}{ Coasting beam } & \multicolumn{2}{|c|}{ Bunched beam } \\
\hline & & $Q_{x}$ & & $Q_{x}$ \\
\hline Low intensity & $\sim 0.8 \times 10^{9}$ & & $\sim 0.4 \times 10^{9}$ & \\
\hline$(\delta p / p)_{\mathrm{rms}}$ & $4.8 \times 10^{-4}$ & & $1.3 \times 10^{-3}$ & \\
\hline$\Delta Q_{x, \mathrm{sc} / y, \mathrm{sc}}$ & $-0.008 /-0.011$ & & $-0.01 /-0.015$ & \\
\hline$\left(I_{1} / I_{0}\right)_{\min }$ & 0.26 & 4.3325 & 0.31 & 4.3325 \\
\hline$\left(\epsilon_{1} / \epsilon_{0}\right)_{\max }$ & 1.20 & 4.3365 & 1.21 & 4.3385 \\
\hline$z_{1} / z_{0}$ & & & 0.79 & 4.3325 \\
\hline High intensity & $\sim 3.0 \times 10^{9}$ & & $\sim 1.2 \times 10^{9}$ & \\
\hline$(\delta p / p)_{\mathrm{rms}}$ & $4.8 \times 10^{-4}$ & & $1.3 \times 10^{-3}$ & \\
\hline$\Delta Q_{x, \mathrm{sc} / y, \mathrm{sc}}$ & $-0.025 /-0.03$ & & $-0.04 /-0.045$ & \\
\hline$\left(I_{1} / I_{0}\right)_{\min }$ & 0.72 & 4.3365 & 0.23 & 4.3385 \\
\hline$\left(\epsilon_{1} / \epsilon_{0}\right)_{\max }$ & 1.28 & 4.3425 & 1.79 & 4.3425 \\
\hline$z_{1} / z_{0}$ & & & 0.71 & 4.3365 \\
\hline
\end{tabular}

bunch shortening [19]. This correlation was observed but only for a few bunch profiles, each of them obtained for a different beam "shot." Figure 18(a) shows at $Q_{x}=4.3365$ the time evolution of one bunch during $1 \mathrm{~s}$ storage. The quantities shown are the intensity ratio $I / I_{0}$, horizontal emittance ratio $\epsilon_{x} / \epsilon_{x 0}$, and bunch length ratio $z / z_{0}$. Note the strong correlation maintained between beam loss (red curve) and bunch length (green curve) during the whole storage time. In Fig. 18(b) we show the simulation for the same working point. The simulation starts when the bunch is formed at $t=0.1 \mathrm{~s}$. During the first few turns particles are brought out of the beam core and the rms emittance shows this effect with an increase of up to 1.4. Later, after beam loss becomes substantial, the emittance decreases and its pattern closely follows that of the measurement. The bunch length curve saturates in $0.1 \mathrm{~s}<t<0.2 \mathrm{~s}$ equivalent to 147 synchrotron oscillations, while in the experiment it manifests in a different pattern.

This difference is not easy to explain, and it may be due to a defect in modeling of the nonlinear lattice which gives an acceptable fitting for the coasting beam loss [Fig. 6(a)], but whose uncertainty becomes relevant and is emphasized by the dynamics of the periodic resonance crossing. Also, the self-consistency plays here an essential role. In fact the

TABLE III. In this table we compare the key quantities reported in Table II with the correspondent values found from simulations. The numerical predictions on beam loss are acceptably close to the measured value. Only the emittance growth from simulations for the case of a bunched beam at high intensity exceeds significantly the measured one. This is attributed to the limits of the numerical model of the SIS18 which is an approximation of the real synchrotron. The uncertainties in these parameters are the same as those in Figs. 6, 8, 13, and 14.

\begin{tabular}{lcccc}
\hline \hline & \multicolumn{2}{c}{ Coasting beam } & \multicolumn{2}{c}{ Bunched beam } \\
& Experiment & Simulation & Experiment & Simulation \\
\hline Low intensity & & & & \\
$\Delta Q_{x, \mathrm{sc} / y, \mathrm{sc}}$ & $-0.008 /-0.011$ & & $-0.01 /-0.015$ & \\
$\left(I_{1} / I_{0}\right)_{\min }$ & 0.26 & 0.14 & 0.31 & 0.36 \\
$\left(\epsilon_{1} / \epsilon_{0}\right)_{\max }$ & 1.20 & 1.70 & 1.21 & 1.60 \\
$z_{1} / z_{0}$ & & & 0.79 & 0.78 \\
High intensity & & & & \\
$\Delta Q_{x, \text { sc } / y, \text { sc }}$ & $-0.025 /-0.03$ & & $-0.04 /-0.045$ & 0.51 \\
$\left(I_{1} / I_{0}\right)_{\min }$ & 0.72 & 0.84 & 0.23 & 2.44 \\
$\left(\epsilon_{1} / \epsilon_{0}\right)_{\max }$ & 1.28 & 1.52 & 1.79 & 0.81 \\
$z_{1} / z_{0}$ & & & 0.71 & \\
\hline \hline
\end{tabular}



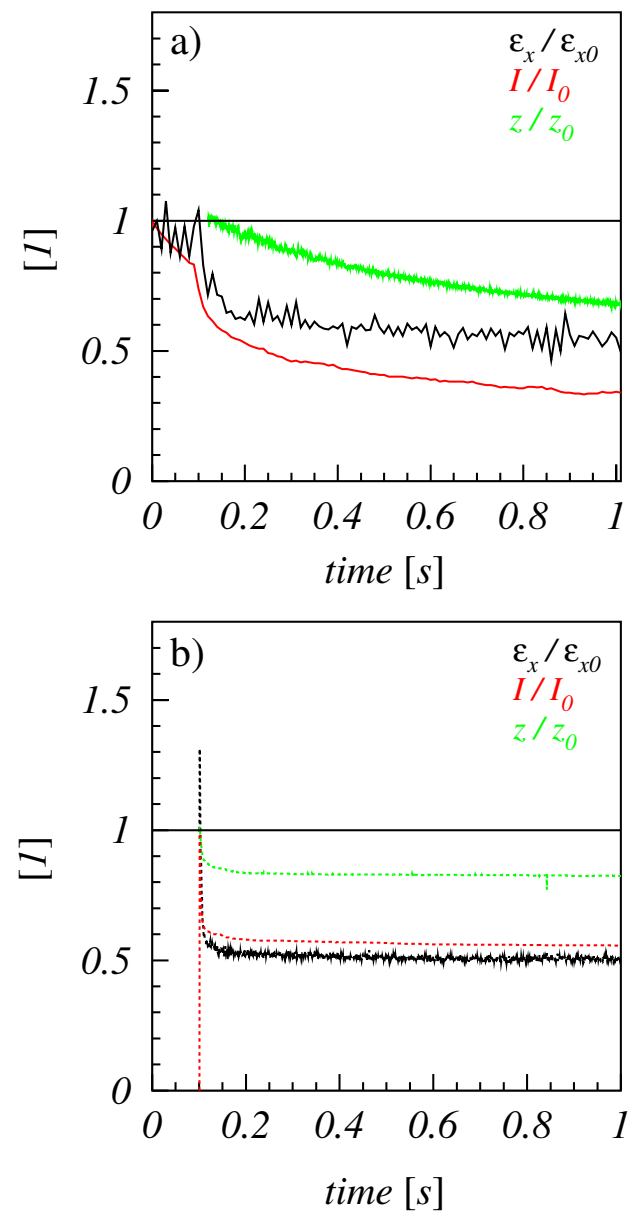

FIG. 18. High intensity bunched beam at $Q_{x}=4.3365$. (a) Transverse-longitudinal beam response to the long-term storage. (b) Simulation results for the same working point. The error bars in these measurements (here not shown) are the same as those in Fig. 14.

final beam survival in the experiment is $33 \%^{1}$ while in the simulation it is $\sim 52 \%$. This difference can be attributed to the missing self-consistency of the space charge calculation, which in the simulation fails to bring new particles to cross the resonance. In fact, with frozen space charge the bunch particles subjected to the periodic resonance crossing are those populating the external "shell" of the longitudinal phase space. Each of these particles is characterized by a single particle tune $Q_{x \text {,part }}$ satisfying the condition $Q_{x \text {,part }}=Q_{x}-\Delta Q_{x}(0)<Q_{x, \text { res }}$ at $z=0$, and $Q_{x \text {,part }}=Q_{x}-\Delta Q_{x}\left(z_{\max }\right)>Q_{x, \text { res }}$ at $z=z_{\max }$, where $\Delta Q_{x}(z)$ is the space charge tune shift of the frozen system for a specific bunch particle as a function of the longitudinal position $z$. The inclusion of the beam loss on the space charge algorithms shrinks the tune spread, and so

\footnotetext{
${ }^{1}$ Note that $33 \%$ is relative to Fig. 6(a), which is obtained for $Q_{x}=4.3365$, not to be confused with the values given in Tables II and III, which refer to the different working point $Q_{x}=$ 4.3385 .
}

particles that before were always below the resonance, may now reach the condition of periodic resonance crossing because of the smaller tune spread. The transverse tune of those bunch particles may now be $Q_{x \text {,part }}>Q_{x \text {,res }}$ when they are at the maximum longitudinal amplitude. This argument suggests that a nonupdating of the space charge with beam loss may be responsible for the weaker beam loss and the flatness of the beam survival from 0.2 to $1 \mathrm{~s}$ in Fig. 18(b). Further studies are required to validate this interpretation of the experimental results.

The correlation between experimental beam loss and bunch shrinkage is made clearer in Fig. 19 by plotting bunch intensity versus bunch length. As pointed out in Ref. [39], a correlation between beam loss and bunch length shrinking is to be expected if the main mechanism leading to beam loss includes a functional dependence on the longitudinal excursion of particles - created here by space charge. The findings of Fig. 19 retrieve the results found in the PS experiment [39] and are consistent with the interpretation that particles with large synchrotron amplitude [see Fig. 17(b)] are lost because they are mainly scattered by the stable islands. The dashed curves in Fig. 19 show what type of correlation may be expected according to the type of longitudinal beam distribution without including the effect of the self-consistency. This comparison reveals that initially the correlation bunch-intensity bunch length falls much faster than is the case for any of the plotted distributions. In this small beam loss regime, the periodic crossing of the resonance is mainly determined by the initial incoherent tune spread. Instead later, the correlation becomes parallel to the KV-like longitudinal distribution (black dashed curve). This pattern reveals a higher complexity which stems from the re-adjustment of the beam distribution due to beam loss and self-consistency effects. It is

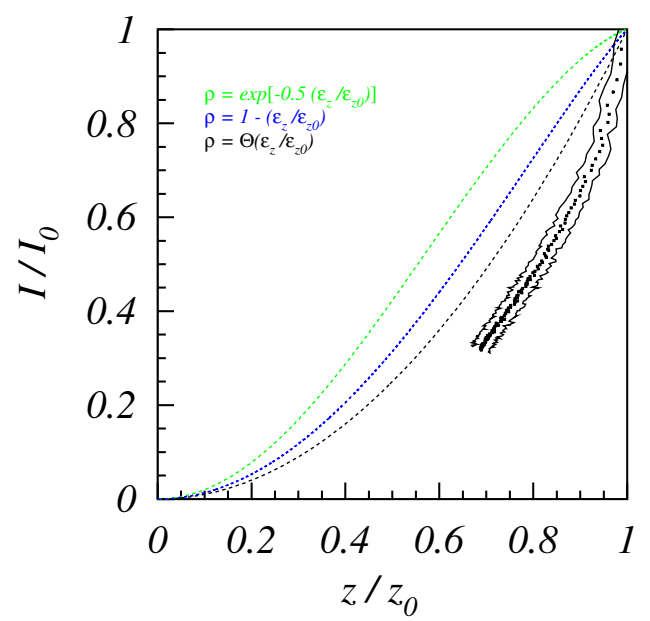

FIG. 19. High intensity bunched beam: beam loss/bunch length correlation. The two continuous thin lines represent the error bar. The dashed curves represent the theoretical correlation for several longitudinal particle distributions: Gaussian (green), Waterbag (blue), and KV (black). 
however surprising that, in spite of the lack of selfconsistency, the experimental curve is almost parallel to the KV-like or waterbag-like theoretical curves.

\section{Trapping or scattering?}

It is relevant to address the question of whether the dynamics here studied is of trapping or of scattering. In order to answer to this question we computed the
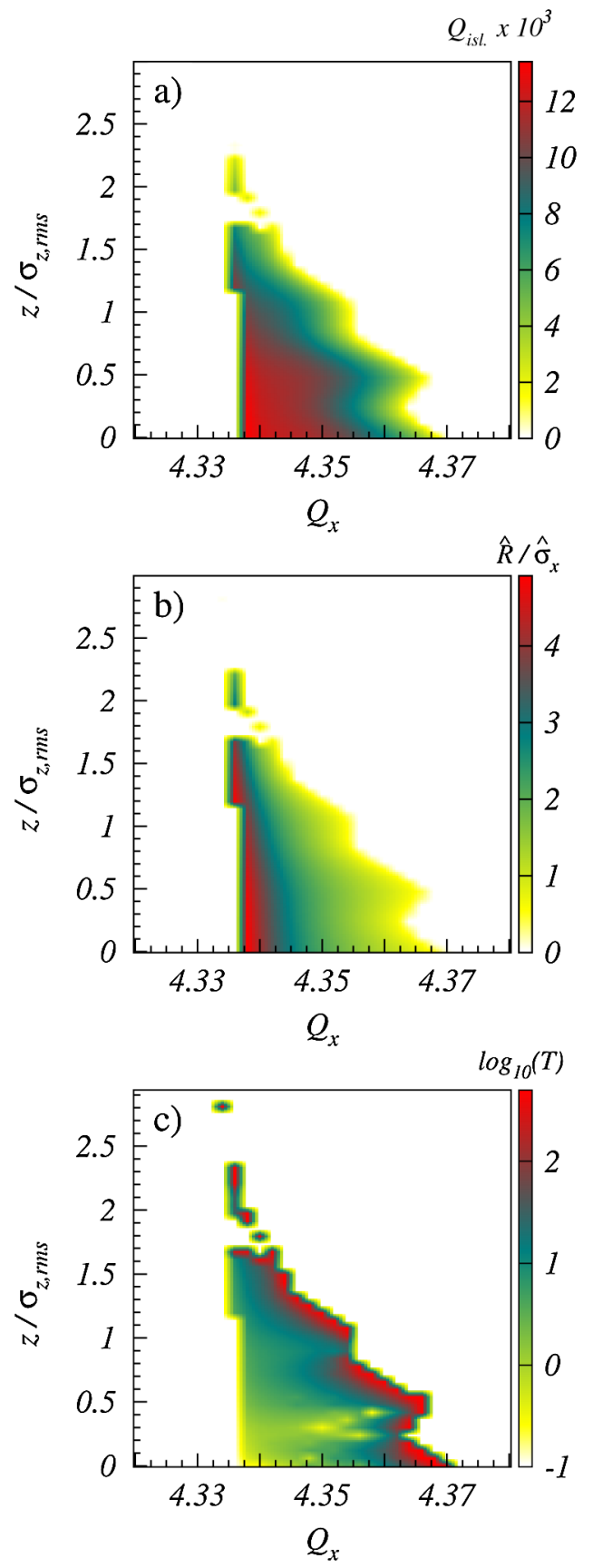

FIG. 20. Main feature characterizing the periodic resonance crossing: (a) Island tune vs $z$, and $Q_{x}$. (b) Fixed point radius (in CS) vs $z$, and $Q_{x}$. (c) Adiabaticity parameter $T$ vs $z$, and $Q_{x}$. In the white areas the fixed points do not exist. parameter $T$, which is the ratio of the speed of migration of the fixed points to the maximum speed of rotation of the particle in the islands. We compute for several machine tunes $Q_{x}$ the location of the fixed points in the plane $x-p_{x}$ and the island tunes $Q_{x, \text { isl }}$. In these calculations we keep the longitudinal motion frozen so that a particle coordinate $z$ remains constant. In Fig. 20(a) is shown the dependence of the island tune on $\left(Q_{x}, z\right)$. The picture shows that at fixed machine tune $Q_{x, \text { isl }}$ is maximum at $z=0$ decreasing its value for larger $z$ until it reaches zero when the fixed points are close to $x=p_{x}=0$. For $Q_{x}=4.336$ the fixed points disappear for $z \leq 1.2 \sigma_{z}$ as they are located beyond the physical or dynamical aperture here modeled. Figure 20(b) shows the amplitude of the fixed points $\hat{R}=\sqrt{\hat{x}_{f}^{2}+\hat{p}_{x, f}^{2}}$ in Courant-Snyder coordinates as a function of $\left(Q_{x}, z\right)$. This picture clearly shows that the larger amplitude of the fixed points is at $Q_{x} \simeq 4.34$ and $z=0$. During the bunch storage the synchrotron motion leads a bunch particle through several positions $z$, and the instantaneous fixed points span from a maximum value to zero. In Fig. 20(c) it is shown $T$ as a function of $\left(Q_{x}, z\right)$. Note that $T \propto Q_{s}$ is computed for the synchrotron tune of $Q_{s}=0.0069$. If $T \ll 1$ the island crossing happens in an adiabatic condition and the particle can remain trapped in it. This picture shows that when the fixed points are close to the origin $T>1$ (red region) and that for $z$ closer to the bunch center and $Q_{x}$ close the $3 \mathrm{rd}$ order resonance $T$ is smaller than 1 (yellow region near $Q_{x}=4.34$, and $z=0$ ).

It is therefore expected that particles with small transverse amplitudes are crossed by islands which cannot trap but rather create a scattering.

When islands cross particles at large transverse amplitudes, they are characterized by a smaller $T$ enhancing the probability of trapping. However, this process happens only for particles that already are at large amplitudes. The overall bunch dynamics is then characterized by particles which are subjected by a different level of scattering almost reaching a trapping condition.

\section{CONCLUSION}

In this experiment we have studied the interplay between the transverse space charge of a bunched beam and a 3rd order lattice resonance. The nonlinear dynamics differ in this case from that in the preceding CERN-PS experiment. In the latter, an octupole driven 4th order resonance always creates stable islands, even in the presence of very weak space charge. When the space charge tune spread is larger than the distance of the machine tune from the resonance, it directly determines the position of the fixed points.

Alternatively, for a 3rd order resonance, in the absence of space charge there are no stable islands but rather a triangular separatrix which divides the phase space into regions of stable and unstable beam dynamics. The nonlinear dynamics is here substantially affected by the space 
charge, which breaks the unstable region and creates three stable islands and at the same time sets the location of the fixed points (as shown in Figs. 7 and 9).

Therefore, in spite of the intrinsic differences between 3rd and 4th order resonances, space charge leads to similar patterns in the beam response. This allows us to interpret on a solid base that in both experiments (PS, and SIS18) the underlying beam physics is the same. In particular, from our experimental findings in which we compared several beams, coasting and bunched, at different intensities, we find a strong evidence that the measured global quantities as emittance ratio and beam loss are interpretable mainly in terms of scattering effects induced by space charge, previously demonstrated only via numerical and analytical methods. Trapping effects are mitigated by a speed of the synchrotron motion, which prevents an adiabatic regime of beam particles within the 3rd order islands. The comparison of the code predictions with the experimental results has given reasonably good agreement in spite of the limited knowledge of the SIS18 synchrotron, which does not allow a complete simulation of the real experimental conditions. The issue of self-consistency in the presence of a large relative beam loss, which feeds back to the beam distribution, will be addressed in future studies.

We add here that our experimental findings also strengthen the understanding of the electron cloud incoherent effects $[40,41]$ where the essential mechanism suggesting a similarity with space charge is the correlation of the amount of electron pinch with the extent of the bunch that has passed through the electron cloud. The similarities with the space charge are discussed in Ref. [42].

\section{ACKNOWLEDGMENTS}

We thank the support of O. Boine-Frankenheim, and P. Spiller. One the authors (O. C.) thanks K.-P. Ningel and M. Mehler for help in setting the external SIS18 RF cycle. G. F. is indebted to C.-K. Wetzel for the accelerator settings and S. Appel for the discussions on momentum spread.

\section{APPENDIX A}

We derive here the relation between beam loss for a low intensity Gaussian beam and the distance of the machine tune $Q_{x}$ from a 3 rd order resonance excited by one sextupole in a linear lattice. We start by observing that near the 3rd order resonance the separatrix assumes a triangular shape with straight sides. The radial distance $\hat{a}$ (in Courant-Snyder coordinates) of the fixed point $(\hat{a}, 0)$ is given by [43]

$$
\hat{a}=-\frac{16 \pi}{\beta_{x}^{3 / 2} K_{2}} \Delta Q_{x},
$$

where $\beta_{x}$ is the beta function at the location of the sextupole, $K_{2}$ is its integrated strength, and $\Delta Q_{x}$ is the distance from the third order resonance. Note that $\hat{a}$ can be positive or negative, which simply means that the triangular separatrix may be mirrored on the $\hat{x}^{\prime}$ axis. The area $A_{x}$ within the separatrix is given by

$$
A_{x}=3 \sqrt{3} \frac{64 \pi^{2}}{\beta_{x}^{3} K_{2}^{2}} \Delta Q_{x}^{2} .
$$

Consider a 1D uniform beam of $N_{0}$ particles and edge emittance $\epsilon_{x}$ matched with the linear optics. The area of the phase space occupied by the beam is $\pi \epsilon_{x}$, that is the particle density is $\rho=N_{0} /\left(\pi \epsilon_{x}\right)$. When the distance from the 3rd order resonance $\Delta Q_{x}$ is small, the beam survival to long-term storage is given by the number of particles found inside the separatrix which is $N=\rho A_{x}$, equal to

$$
\frac{N}{N_{0}}=\frac{1}{\epsilon_{x}} 3 \sqrt{3} \frac{64 \pi}{\beta_{x}^{3} K_{2}^{2}} \Delta Q_{x}^{2}
$$

In case mechanical aperture limits are present, the dependence of asymptotic beam loss on $\Delta Q_{x}$ changes if the radius of the fixed points $\hat{a}$ exceeds the radius of the acceptance $\hat{a}_{m}$. The transition to this new beam loss regime occurs when the fixed points touch the acceptance. When this happens the beam survival is $A / A_{m}$, where $A_{m}$ is the phase space area of the linear acceptance, i.e. $A_{m}=\pi \hat{a}_{m}^{2}$, and $A$ is the area within the $1 \mathrm{D}$ separatrix $A=(3 \sqrt{3} / 4) \hat{a}_{m}^{2}$. The beam survival at this transition point is therefore

$$
\frac{N}{N_{0}}=\frac{A}{A_{m}}=\frac{3 \sqrt{3}}{4} \frac{1}{\pi}=41.3 \%
$$

We benchmark Eq. (A3) and the transition threshold in Eq. (A4) via simulations. We take a model of the SIS18 lattice with acceptances $A_{x / y}=151 / 87 \mathrm{~mm} \mathrm{mrad}$, and apply between two synchrotron periods one normal sextupole of integrated strength $K_{2}=0.05 \mathrm{~m}^{-2}$ : At this position the beta function is $\beta_{x}=12.71 \mathrm{~m}$. We then inject a matched $\mathrm{KV}$ beam of $10^{4}$ macroparticles with emittances $\epsilon_{x}=$ $200 \mathrm{~mm} \mathrm{mrad}, \epsilon_{y}=0.01 \mathrm{~mm} \mathrm{mrad}$ and track it for $10^{4}$ turns. In Fig. 21(a) we show the beam survival versus $\Delta Q_{x}$ and find a good agreement between the theoretical prediction of Eq. (A3) (red) and the numerical results (black). Note the discrepancy of the prediction arising when the fixed points exceed the mechanical aperture. This happens when the beam survival is larger than $41 \%$ as predicted by Eq. (A4).

The beam loss analysis for a Gaussian beam is more complicated. We note first that, for a matched 1D Gaussian beam with rms emittance $\tilde{\boldsymbol{\epsilon}}_{x}$, the survived particles when removing the particles located at radius larger than $\hat{r}_{c}$ is given by the formula $N / N_{0}=1-e^{-\hat{r}_{c}^{2} /\left(2 \tilde{\epsilon}_{x}\right)}$. From the geometry of the separatrix we distinguish between the maximum radius of the fixed points $\hat{a}$, and the radius $\hat{a}^{\prime}=\hat{a} / 2$ of the circle inscribed into the triangle formed by the separatrix. The beam survival for a cut at $\hat{a}$ and $\hat{a}^{\prime}$ 
overestimates/underestimates the beam survival due to the real separatrix, which is clearly in between the two extremes, that is

$$
1-e^{-\hat{a}^{\prime 2} /\left(2 \tilde{\epsilon}_{x}\right)}<\frac{N}{N_{0}}<1-e^{-\hat{a}^{2} /\left(2 \tilde{\epsilon}_{x}\right)} .
$$

Clearly, given the geometry of the problem the beam survival depends only on the ratio $\hat{a}^{2} / \tilde{\epsilon}_{x}$, and from Eq. (A5) we search for a solution of the form

$$
\frac{N}{N_{0}}=1-e^{-\sum_{n=1}^{\infty} \alpha_{n}\left(\hat{a}^{2} / \tilde{\epsilon}_{x}\right)^{n}}
$$

The term $\alpha_{1}$ is computed in the limit of small distances from the resonance. In fact when $\Delta Q_{x}$ is small, the separatrix $\hat{a}$ is small and Eq. (A6) becomes

$$
\frac{N}{N_{0}}=\alpha_{1} \frac{\hat{a}^{2}}{\tilde{\boldsymbol{\epsilon}}_{x}}
$$

The particle distribution within the small separatrix is now almost uniform $\rho_{u}$ and therefore we may compute the surviving particles as $N=\rho_{u} A_{x}$. As the Gaussian distribution is described by

$$
\rho=\frac{N_{0}}{2 \pi \tilde{\boldsymbol{\epsilon}}_{x}} e^{-\hat{r}^{2} /\left(2 \tilde{\boldsymbol{\epsilon}}_{x}\right)}
$$

for $\hat{r} \rightarrow 0$, we find $\rho_{u}=N_{0} /\left(2 \pi \tilde{\epsilon}_{x}\right)$; therefore the surviving particles are

$$
\frac{N}{N_{0}}=\frac{3 \sqrt{3}}{8 \pi} \frac{\hat{a}^{2}}{\tilde{\epsilon}_{x}}
$$

Comparing this formula with Eq. (A7), we find that

$$
\alpha_{1}=\frac{3 \sqrt{3}}{8 \pi} .
$$

The other coefficients of the expansion in Eq. (A6) may be computed via Monte Carlo integration. In Fig. 21(b) the beam survival for several ratios $\hat{a}^{2} / \tilde{\epsilon}_{x}$ is shown. The red curve is obtained using Eq. (A6) with $\alpha_{2}=-3.2 \times 10^{-3}$, $\alpha_{3}=0.6 \times 10^{-4}, \alpha_{4}=-3.7 \times 10^{-7}$, and $\alpha_{5}=10^{-9}$. The good fit with the numerical findings shows that using the first five terms of the expansion in Eq. (A6) is an acceptable approximation for practical use.

We then benchmarked the predictions of Eq. (A6) (here $\hat{a}$ is now expressed as function of $\Delta Q_{x}$ ) with multiparticle simulations. The results are shown in Fig. 21(c) for a Gaussian beam with rms emittance $\tilde{\boldsymbol{\epsilon}}_{x / y}=$ 10/0.01 $\mathrm{mm}$ mrad (i.e., a 1D beam). The beam distribution has $10^{4}$ macroparticles and is tracked for $10^{4}$ turns in SIS18. The values of $\beta_{x}$ and the strength of the sextupole are the same as for the KV simulation. Note that in this simulation the relevant part of the beam tails is inside the machine acceptance. Therefore the beam survival far from the resonance is $100 \%$.
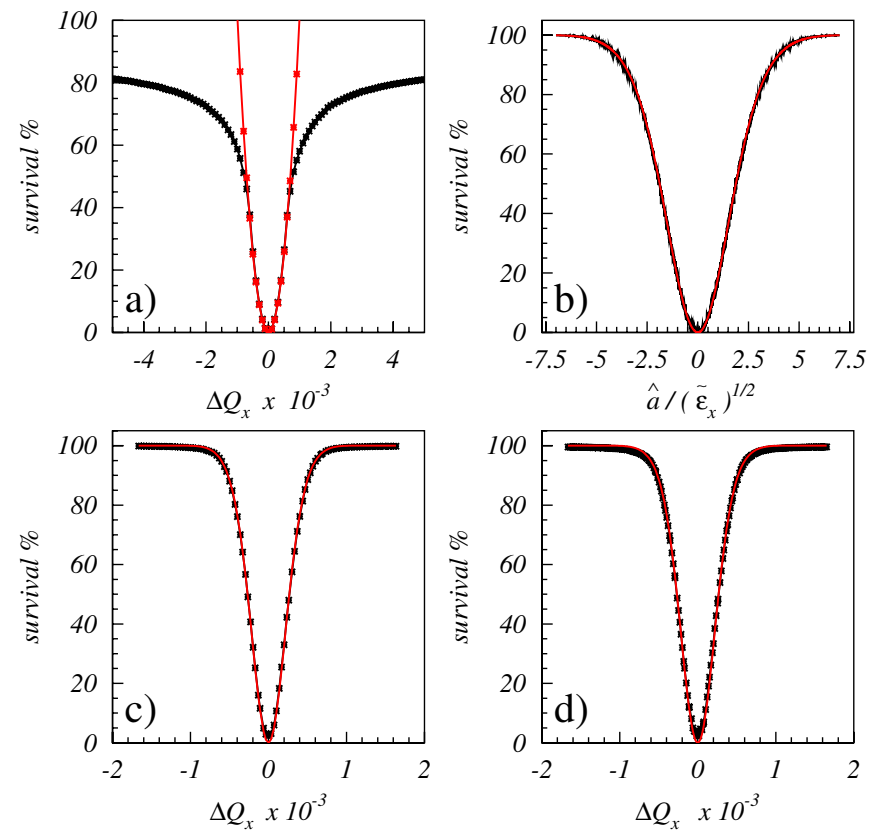

FIG. 21. (a) In black markers are plotting the tracking simulation results for a uniform beam distribution. In red markers are plotting the analytic results from Eq. (A3). At efficiency of $41 \%$ the fixed points intercept the acceptance. From there on the formula exhibits large discrepancy with respect to the numerical findings. (b) Beam survival for a 1D Gaussian beam distribution as a function of $\hat{a}^{2} / \tilde{\boldsymbol{\epsilon}}_{x}$. (c) Beam survival for a 1D Gaussian beam distribution as a function of $\Delta Q_{x}$ (black), and theoretical prediction (red). (d) Beam survival for a 2D Gaussian beam distribution as function of $\Delta Q_{x}$ (black), and theoretical prediction for a $2 \mathrm{D}$ beam (red).

We also checked the robustness of Eq. (A6) for a 2D Gaussian beam by repeating the same simulation as for the 1D Gaussian beam but now for the emittances $\tilde{\boldsymbol{\epsilon}}_{x / y}=$ 10/7 mm mrad. The numerical results, shown in Fig. 21(d), confirm the applicability of Eq. (A6) to a two-dimensional beam. We therefore use Eq. (A6) to predict the beam loss stop band $\Delta Q_{x, \mathrm{sb}}$ defined by an edge where beam loss is $1 \%$. By imposing this condition in Eq. (A6) we find

$$
\Delta Q_{x, \mathrm{sb}}=0.217 \sqrt{\tilde{\epsilon}_{x}} \beta_{x}^{3 / 2}\left|K_{2}\right| .
$$

This equation has been used in Sec. III A for setting the sextupole strength to match the experimental beam loss stop band.

\section{Effect of an additional octupole}

When several types of nonlinearities are simultaneously present, their combined effect on the beam dynamics is difficult to assess. However, the presence of an additional octupole, to the sextupole, does not change the resonance driving term, but rather creates another source of detuning. We add here an extra octupole in the same location of the sextupole. The fixed points are identified by the condition that the total phase advance in three turns is $2 \pi$, that is, 


$$
\frac{1}{2} \beta_{x}^{2} K_{3} \hat{a}^{2}+\beta_{x}^{3 / 2} K_{2} \hat{a}+16 \pi \Delta Q_{x}=0
$$

For a 1D KV distribution the beam survival will be then expressed as

$$
\frac{1}{2} \beta_{x}^{2} K_{3} \epsilon_{x} \lambda \frac{N}{N_{0}} \pm \beta_{x}^{3 / 2} K_{2} \sqrt{\epsilon_{x} \lambda \frac{N}{N_{0}}}+16 \pi \Delta Q_{x}=0
$$

with $\lambda=4 \pi /(3 \sqrt{3})$. The sign \pm should be chosen according to whether the separatrix is inverted or not, that is, according to the sign of $\hat{a}$. We benchmarked this formula and present the results in Fig. 22(a) where we consider a sextupole with $K_{2}=0.1 \mathrm{~m}^{-2}$ and an octupole with $K_{3}=$ $-3 \mathrm{~m}^{-3}$. The case for the 1D Gaussian beam is shown in Fig. 22(b) [for this picture and Fig. 22(c) we take $K_{3}=$ $-1 \mathrm{~m}^{-3}$ ]. Here given $\Delta Q_{x}, \hat{a}$ is found from Eq. (A12), and the beam survival is found from Eq. (A6). The beam survival prediction is excellent. The robustness of these predictions for the 2D Gaussian beam is shown in Fig. 22 (c), the emittances are again $\epsilon_{x / y}=10 / 7 \mathrm{~mm}$ mrad. Note that in all these pictures the effect of the octupole is to "tilt" the beam survival curve which becomes asymmetric with respect to the center of the resonance. This effect has been used to create the proper asymmetry in the beam loss in Fig. 6(b) with an octupole.
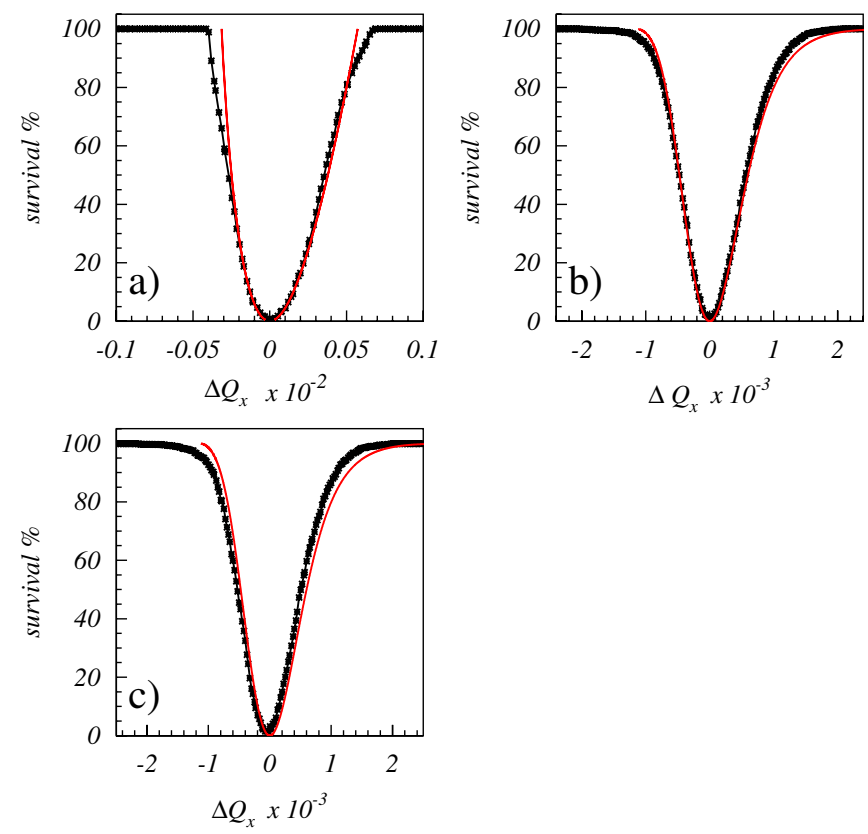

FIG. 22. Case of one sextupole and one octupole: (a) Beam survival for a $1 \mathrm{D} \mathrm{KV}$ beam distribution as a function of $\Delta Q_{x}$ (black), and theoretical prediction (red). (b) Beam survival for a 1D Gaussian beam distribution as a function of $\Delta Q_{x}$ (black), and theoretical prediction (red). (c) Beam survival for a 2D Gaussian beam distribution as a function of $\Delta Q_{x}$ (black), and theoretical prediction (red).

\section{APPENDIX B}

In this Appendix the procedures for assigning the error bars are discussed. The error bars here represent 1 standard deviation.

\section{Error bar in the transverse rms size}

We present here the analysis of the error bar attributed to the rms size computed from the IPM data. In Fig. 23 we plot a schematic of the IPM measurement discretization. The device is equipped with 64 wires equally spaced by $\Delta x=2.1 \mathrm{~mm}$. The $i$ th wire detects a signal proportional to the beam density projection in the interval $\Delta x(i-1 / 2)<$ $x<\Delta x(i+1 / 2)$ corresponding to the blue area in the example of Fig. 23. The signal is then amplified and digitized obtaining as output an integer number $N_{i}$ associated to the $i$ th wire. Each data $N_{i}$ will be subjected to an error $\delta N_{i}$, which is the same for all wires. The effective measured value is then $N_{m, i}=N_{i}+\delta N_{i}$. It should also be taken into account that the position of the beam is typically not centered on the geometrical center of the IPM: In Fig. 23 this shift is indicated with $\Delta x_{p}$. In order to avoid a spurious signal, we cut the data from wires with $N_{i}$ less than $N_{c}=\lambda_{c} N_{i, \max }$ with $\lambda_{c}=0.1$, i.e., the lower $10 \%$ of the maximum of the beam distribution. The rms beam size is computed from the measured data as $\tilde{x}=\sqrt{\left\langle x^{2}\right\rangle-\langle x\rangle^{2}}$ from the moments

$$
\begin{aligned}
\left\langle x^{2}\right\rangle & =\frac{\sum_{i}^{*}(i \Delta x)^{2} N_{m, i}}{\sum_{i}^{*} N_{m, i}}, \\
\langle x\rangle & =\frac{\sum_{i}^{*}(i \Delta x) N_{m, i}}{\sum_{i}^{*} N_{m, i}},
\end{aligned}
$$

where the symbol $\sum_{i}^{*}$ refers to a sum of the cut data. Note that these moments depend on $\Delta x_{p}, N_{c}, \delta N_{i}$, and that the variation of $\Delta x_{p}$ should be considered in the interval $0<\Delta x_{p}<\Delta x$ since larger values are redundant. Based on this IPM modeling, in order to assess the effect of the

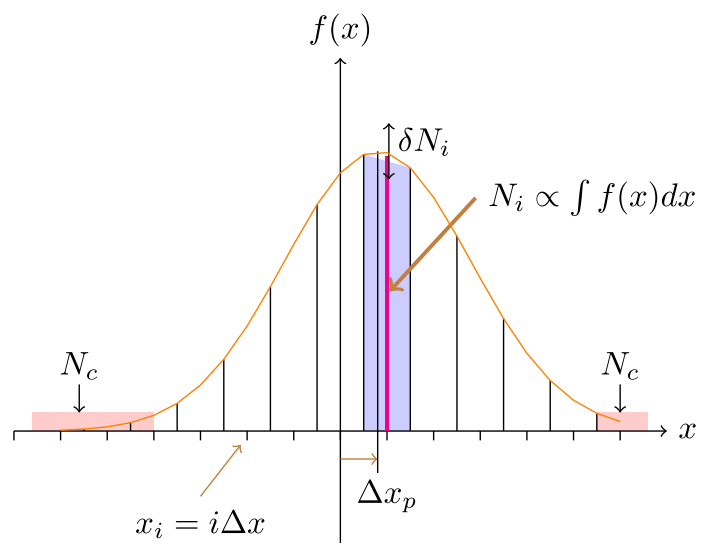

FIG. 23. Schematic of the IPM acquisition, errors and selection of the measured data. 
discretization, we have performed a numerical study. The key quantity is the number of wires per beam sigma $B=$ $\sigma_{x} / \Delta x$ (here $\sigma_{x}$ is expressed in $\mathrm{mm}$ ). Clearly we expect large deviations when $B<1$ as the beam is too small for the resolution of the device. We consider several $\Delta x_{p}$, $0.06<\lambda_{c}<0.15$, for $\delta N_{i}=0.02 \times \max \left\{N_{i}\right\}$. In Fig. 24 (a) we show the calculated average rms size versus $B$, the blue curve is obtained by an analytic fit which we find to be

$$
\frac{\langle\tilde{x}\rangle}{\sigma_{x}}=1-\lambda_{c} 0.9+\frac{0.041}{B^{2}} .
$$

Figure 24(b) shows the standard deviation of the distribution of rms sizes. Again the blue curve is obtained by the analytical fit

$$
\frac{\delta_{\tilde{x}}}{\sigma_{x}}=\frac{0.043}{B}+0.33 \delta N_{i}
$$

The interpretation of the two analytical fits is as follows. In Eq. (B3) the term $1-\lambda_{c} 0.9$ includes the effect of the cut off $\lambda_{c}$, which reduces the effective rms bunch size. The last term includes the effect of the discretization. In Eq. (B4) the term $0.043 / B$ represents the average effect deriving from the shift of the beam distribution, which depends on $\Delta x_{p}$. The last term includes the contribution of the error bar associated to each wire.

The practical procedure in the experiment was to repeat several times the measurement for the same working point. In this process the fluctuations of the ion source also plays a role because from shot to shot beam intensity as well as a beam size variation is observed. We take these effects into account by treating the data as follows: (1) Several measurements are taken from the same working point. (2) For each measurement we compute the rms size with Eqs. (B1) and (B2), correct the systematic error with Eq. (B3), and assign the error bar using Eq. (B4). These quantities are then converted, for the $j$ th measurement, into the emittance $\epsilon_{x, j}$, and emittance error bar $\delta \epsilon_{x, j}$ using the beta function at the location of the IPM. (3) We then calculate the average emittance $\epsilon_{a v, x}$, and the emittance error bar $\Delta \epsilon_{a v, x}$ from the collection of all $\epsilon_{x, j}, \delta \epsilon_{x, j}$ for each repeated measurement $j$ with
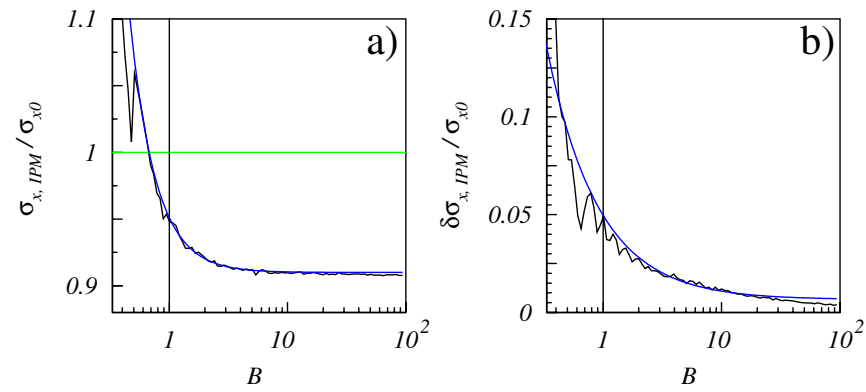

FIG. 24. Study of the systematic and random error for $\lambda_{c}=$ 0.1 , and $\delta N_{i}=0.02 \delta N_{i, \max }$. (a) Systematic shift of the rms size of a Gaussian distribution. (b) The rms error of the rms size. Here we identified $\sigma_{x, \text { IPM }}$ with $\langle\tilde{x}\rangle$ and $\delta \sigma_{x, \text { IPM }}$ with $\delta \tilde{x}$.

$$
\epsilon_{\mathrm{av}, x}=\left\langle\epsilon_{x}\right\rangle_{j}, \quad \Delta \epsilon_{\mathrm{av}, x}=\sqrt{\left\langle\epsilon_{x}^{2}\right\rangle_{j}-\left\langle\epsilon_{x}\right\rangle_{j}^{2}+\left\langle\delta \epsilon_{x}^{2}\right\rangle_{j}},
$$

where $\langle\cdot\rangle_{j}$ is an average over the repeated measurements. By doing so the error bar of the rms emittance is enlarged as it includes the fluctuation of the ion source. (4) The error on the emittance ratio is then computed via a standard propagation of the error obtaining the error bars plotted in all the pictures reporting measurements covered in this paper.

\section{Error bar in the longitudinal rms size}

The calculation of the longitudinal rms beam size follows a similar procedure as described in the previous section. The main difference lies in the fact that the number of "wires" per beam sigma is now quite large $(B \simeq 100)$. The error in the measurements is mainly determined by the oscilloscope resolution which is $17.9 \mathrm{mV}$. Another source of error is in the beam profile fluctuations which are visible when looking at the beam profile time evolution. As the number of longitudinal beam profiles during the storage time of $1 \mathrm{~s}$ is $\sim 4000$, we evaluate the error bar for each density acquisition by taking 30 consecutive beam profiles. From these profiles we obtain an average beam profile and the variance is the error bar. In Fig. 25 we show an example of the average beam profile and the error bar associated to the longitudinal charge line density. In order to find the average rms longitudinal length $\sigma_{z}$ and the error bar $\delta \sigma_{z}$, we have made a numerical study of the propagation of the errors on the rms beam size. Similarly, applying the procedure adopted for the transverse data, we cut the longitudinal data now to $20 \%$ as the error bar is now $\sim 5 \%$ of the maximum of the profile (see Fig. 25) and compute the rms size. This value is then corrected from the systematic error using Eq. (B3) with $B \sim 100$. The procedure to assign the

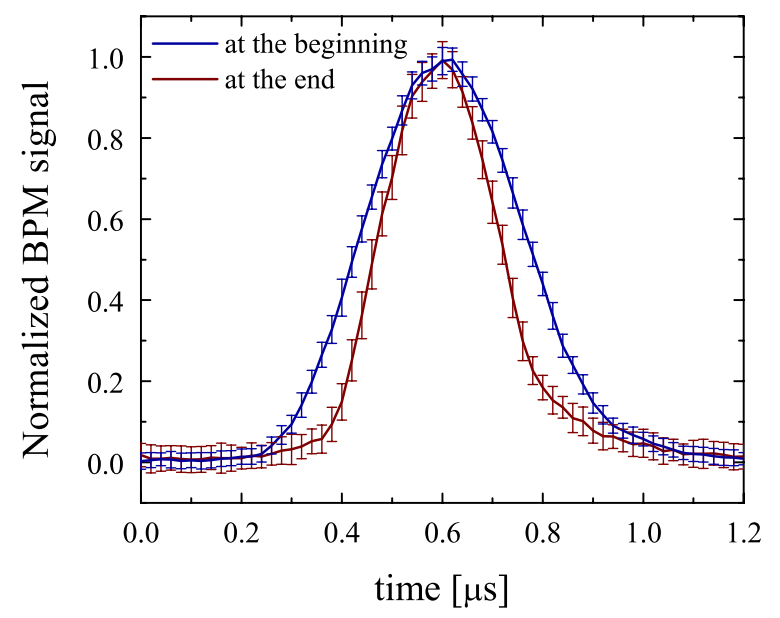

FIG. 25. Example of average longitudinal beam profile obtained from 30 consecutive profiles for the measurement at $Q_{x}=$ 4.3365 with the bunched beam at high intensity. 
error bar to the rms size follows the same procedure as for obtaining Fig. 24(b).

\section{Error bar in the beam intensity measurements}

The measurement of the beam intensity is performed via the IPM, which in conjunction with the beam profile data stores also an uncalibrated signal $\mathcal{S}$ proportional to the beam current $I$ via $I=\alpha \mathcal{S}$. This signal is calibrated with the signal measured from the current transformer which has a negligible error bar. In fact for the dc transformer, one can make the following error estimation: The error for the analog signal is better than $2 \times 10^{-3}$ of the full-scale value. In addition, there is an absolute error of about $5 \mu \mathrm{A}$ due to offset drifts and accuracy of the analog electronics. This is only important for the lower full-scale ranges $300 \mu \mathrm{A}$ and $1 \mathrm{~mA}$. As the full scale in our measurements is in the range $0-30 \mathrm{~mA}$, only the analog error is relevant which gives an error bar of $\pm 60 \mu \mathrm{A}$, the same throughout all four campaigns, and is not included in the pictures of the manuscript. The discretization, operated by the IPM, of the signal $\mathcal{S}$ is taken to be $\delta \mathcal{S}=10$ (arbitrary units) which corresponds to a relative error ranging from $10 \%$ for low beam intensity to $2 \%$ for high beam intensity. The average error bar plotted into the results of this paper is obtained with the formula

$$
I_{\mathrm{av}}=\langle I\rangle_{j}, \quad \Delta I_{\mathrm{av}}=\sqrt{\left\langle I^{2}\right\rangle_{j}-\langle I\rangle_{j}^{2}+\left\langle\delta I^{2}\right\rangle_{j}}
$$

The index $j$ refers to the $j$ th repeated measurement. Again, this procedure allows one to take into account repeated measurements enlarging the error bars.

\section{Error bar in the machine tunes}

From previous experience reported in Refs. [25,26], a systematic tune shift affects the tune values set into the SIS18 control system. As discussed in Sec. III A 3, we have found the systematic shift in the tune by finding the location of the 3rd order resonance. This shift has been used to correct the systematic error created by the SIS18 control system. The error in this procedure is due to the distance of two consecutive tunes in Fig. 6(a), which is $2 \times 10^{-3}$. It is straightforward to show that the standard deviation of the set tune is 0.29 times the distance of discretization among tunes. Therefore we assign to the tunes an error bar of $6 \times 10^{-4}$. As far as concerns the random component on the tune, experiences from other studies in SIS18, as for the nonlinear tune response matrix [29], allows one to assign a standard deviation due machine fluctuation of $\delta Q_{x}=5 \times$ $10^{-5}$ over a measurement time of 6 hours.

Therefore we assign to our tunes an error bar of $6 \times 10^{-4}$, but we do not mark it in the pictures to improve the readability.

\section{Error bar in simulation results}

As our simulations are made with relatively few particles $\left(N_{0}=2000\right)$, we also assign error bars to the simulation results. The estimation of these uncertainties does not reflect the propagation of the initial discretization of the particle distribution to the final distribution at the end of the simulation, but rather provides an estimate of the fluctuation originated by the few particles, which should represent the physical distribution. We assign the error bar as follows.

$I / I_{0}$. - The number of particles at any time during the tracking has a fluctuation

$$
\delta\left(\frac{I}{I_{0}}\right)=\sqrt{\frac{I}{I_{0}}-\left(\frac{I}{I_{0}}\right)^{2}} \frac{1}{\sqrt{N_{0}}} .
$$

$\epsilon_{x} / \epsilon_{x 0}$. - The fluctuation of the rms emittance of a Gaussian distribution is $\delta \epsilon_{x} / \epsilon_{x}=1 / \sqrt{N}$ in good approximation for $N>20$. Therefore

$$
\frac{\delta\left(\epsilon_{x} / \epsilon_{x 0}\right)}{\epsilon_{x} / \epsilon_{x 0}}=\frac{1}{\sqrt{N_{0}}} \sqrt{\frac{1+I / I_{0}}{I / I_{0}}} .
$$

$z_{x} / z_{0}$. - The fluctuation of the rms size emittance of a Gaussian distribution is $\delta\left(z / z_{0}\right)=\delta \sqrt{\epsilon_{z} / \epsilon_{z 0}}=$ $z_{0} /(2 z) \delta\left(\epsilon_{z} / \epsilon_{z 0}\right)$, where the quantity $\delta\left(\epsilon_{z} / \epsilon_{z 0}\right)$ is the same as for Eq. (B7).

[1] G. Guignard, CERN Report No. 78-11, 1978.

[2] X. Huang, S. Y. Lee, E. Prebys, and R. Tomlin, Phys. Rev. ST Accel. Beams 8, 064001 (2005).

[3] S. Y. Lee, Phys. Rev. Lett. 97, 104801 (2006).

[4] I. Hofmann, G. Franchetti, S. Y. Lee, in Proceedings of the 39th ICFA Workshop, Tsukuba, 2006, WEBX05, p. 268.

[5] A. Schoch, CERN Report No. 57-21 (Proton Synchrotron Division), Section 14, 1958.

[6] A. W. Chao and M. Month, Nucl. Instrum. Methods 121, 129 (1974).

[7] A. I. Neishtadt, Sov. J. Plasma Phys. 12, 568 (1986).

[8] A. I. Neishtadt and A. A. Vasiliev, Nucl. Instrum. Methods Phys. Res., Sect. A 561, 158 (2006).

[9] J. L. Tennyson, J. R. Cary, and D. F. Escande, Phys. Rev. Lett. 56, 2117 (1986).

[10] N. Merminga et al., in Proceedings of the First European Particle Accelerator Conference, Rome, Italy, 1988, edited by S. Tazzari (World Scientific, Teaneck, NJ, 1989), p. 791.

[11] T. Satogata et al., Phys. Rev. Lett. 68, 1838 (1992).

[12] S. Y. Lee et al., Phys. Rev. Lett. 67, 3768 (1991).

[13] D. D. Caussyn et al., Phys. Rev. A 46, 7942 (1992).

[14] A. Chao et al., Phys. Rev. Lett. 61, 2752 (1988).

[15] A. Franchi, S. Gilardoni, and M. Giovannozzi, Phys. Rev. ST Accel. Beams 12, 014001 (2009). 
[16] G. Franchetti and I. Hofmann, AIP Conf. Proc. 642, 260 (2002).

[17] G. Franchetti and I. Hofmann, Nucl. Instrum. Methods Phys. Res., Sect. A 561, 195 (2006).

[18] G. Franchetti, I. Hofmann, M. Giovannozzi, M. Martini, and E. Metral, Phys. Rev. ST Accel. Beams 6, 124201 (2003); E. Metral et al., Nucl. Instrum. Methods Phys. Res., Sect. A 561, 257 (2006).

[19] G. Franchetti et al., in Proceedings of the 33rd ICFA Advanced Beam Dynamics Workshop: High Intensity High Brightness Hadron Beams (AIP, New York, 2005), Vol. 773, p. 137.

[20] P. Spiller et al., Nucl. Instrum. Methods Phys. Res., Sect. A 561, 305 (2006); FAIR Technical Design Report SIS100, edited by P. Spiller, GSI, 2008.

[21] P. Spiller, in Proceedings of the 11th European Particle Accelerator Conference, Genoa, 2008 (EPS-AG, Genoa, Italy, 2008), MOPC100, p. 298.

[22] G. Franchetti et al., in Proceedings of the 10th European Particle Accelerator Conference, Edinburgh, Scotland, 2006 (EPS-AG, Edinburgh, Scotland, 2006), p. 2793, THPCH005.

[23] C. Omet, in Proceedings of the 10th European Particle Accelerator Conference, Edinburgh, Scotland, 2006 (Ref. [22]), MOPCH078, p. 211.

[24] T. Giacomini, S. Barabin, P. Forck, D. Liakin, and V. Skachkov, AIP Conf. Proc. 732, 286 (2004).

[25] G. Franchetti, P. Schütt, T. Hoffmann, G. Rumolo, and A. Franchi, Report No. GSI-Acc-Note-2005-02-001.

[26] G. Franchetti, B. Franczak, and P. Schütt, Report No. GSIAcc-Note-2004-05-001.

[27] R. Tomás, M. Bai, R. Calaga, W. Fischer, A. Franchi, and G. Rumolo, Phys. Rev. ST Accel. Beams 8, 024001 (2005).

[28] G. Franchetti, A. Parfenova, and I. Hofmann, Phys. Rev. ST Accel. Beams 11, 094001 (2008).

[29] A. Parfenova and G. Franchetti, in the 23rd Particle Accelerator Conference, 2009, Vancouver, Canada, TH6PFP053 (to be published at the JACoW website).

[30] D.C. Carey, The Optics of Charged Particle Beams (Harwood Academic Publishers, Chur, Switzerland, 1987).
[31] E. Forest, Beam Dynamics A New Attitude and Framework (Harwood Academic Publishers, Chur, Switzerland, 1998).

[32] P. Krejcik, in Proceedings of rhe 1987 IEEE Particle Accelerator Conference, Accelerator Engineering and Technology, 1987 Washington, DC, edited by Eric R. Lindstrom (IEEE, Piscataway, NJ, 1987), p. 1278.

[33] G. Franchetti, I. Hofmann, and G. Turchetti, in Workshop on Space Charge in High Intensity Hadron Rings, Shelter Island, New York, 1998, edited by A. U. Luccio and W. T. Weng, AIP Conf. Proc. No. 448 (AIP, New York, 1998), p. 233

[34] A. Orzhekhovskaya and G. Franchetti, in Proceedings of the ICAP 2006, Chamonix Mont-Blanc, France, edited by Rainer W. Hasse [http://www.jacow.org/], TUPPP05, p. 106.

[35] These formulas are the generalization of K. Schindl, Cern Accelerator School Report No. CAS-CERN, 2003, p. 310. However, we keep here the formalism using the perveance as in M. Reiser, Theory and Design of Charged Particle Beams (Wiley, New York, 1994) p. 236. The formulas Eqs. 4.176, 4.177 in the Reiser book are here extended to a Gaussian beam distribution. Our formulas in case of an axisymmetric beam retrieve Eq. (30) of Schindl at p. 311.

[36] R. Baartman, in Workshop on Space Charge in High Intensity Hadron Rings, Shelter Island, New York, 1998 (Ref. [33]), p. 56.

[37] I. Hofmann, Phys. Rev. E 57, 4713 (1998).

[38] M. Venturini and R. L. Gluckstern, Phys. Rev. ST Accel. Beams 3, 034203 (2000).

[39] G. Franchetti et al., in Proceedings of the 39th ICFA Workshop, Tsukuba, 2006 (Ref. [4]), WEAX01, p. 167.

[40] E. Benedetto, G. Franchetti, and F. Zimmermann, Phys. Rev. Lett. 97, 034801 (2006).

[41] K. Ohmi and K. Oide, Phys. Rev. ST Accel. Beams 10, 014401 (2007).

[42] G. Franchetti, I. Hofmann, W. Fischer, and F. Zimmermann, Phys. Rev. ST Accel. Beams 12, 124401 (2009).

[43] W. Hardt, PS/DL/LEAR Note 81-6, 1981. 